\title{
Combination of Transcranial Magnetic Stimulation with Electromyography and Electroencephalography: Application in Diagnosis of Neuropsychiatric Disorders
}

\author{
Faranak Farzan ${ }^{1}$, Mera S. Barr1, \\ Paul B. Fitzgerald ${ }^{2}$ and Zafiris J. Daskalakis ${ }^{1}$ \\ ${ }^{1}$ Centre for Addiction and Mental Health, \\ University of Toronto, Toronto \\ ${ }^{2}$ Monash Alfred Psychiatry Research Centre, \\ The Alfred and Monash University School of Psychology and Psychiatry, Melbourne \\ ${ }^{1}$ Canada \\ ${ }^{2}$ Australia
}

\section{Introduction}

Transcranial magnetic stimulation (TMS) is a non-invasive brain stimulation technique that allows for in vivo examination of cortical processes. In 1985, Barker et al. introduced TMS as a tool for investigating the functional state of the motor pathways in patients with neurological disorders as well as in healthy human subjects (Barker et al., 1985). It was shown that a single TMS pulse applied to the motor cortex could activate cortical tissues associated with the hand or leg muscles and this activation could elicit a motor evoked potential (MEP) at the periphery captured through electromyography (EMG) recordings. The amplitude, area, latency and duration of this TMS induced MEP could then be used to investigate the integrity of the corticospinal pathways and the activation threshold of human motor cortex. Since this discovery, the combination of single and multi-pulse TMS with peripheral EMG recordings has allowed for examining various other processes in the human motor cortex such as excitability, plasticity, cortico-cortical connectivity, as well as the interaction between excitatory and inhibitory cortical processes. The integration of EMG with TMS has, therefore, offered a valuable tool for assessment of pathological processes that underlie neurological and psychiatric disorders such as Parkinson's disease, dystonia, stroke, Alzheimer's disease, schizophrenia, and depression.

The combination of paired pulse TMS with EMG recordings, for example, permits altering the excitability of the motor cortex and observing the effect of this alteration on subsequent stimulation. In paired pulse TMS, the first TMS pulse (conditioning stimulus) inhibits or facilitates the MEP response to the second TMS pulse (test stimulus). The nature and the strength of this modulatory effect depend on the intensity of the conditioning stimulus and the latency (i.e. interstimulus interval (ISI)) at which it is delivered with respect to the test stimulus. It has been demonstrated that the TMS induced modulation of MEPs are 
selectivity impaired in several neurological conditions (e.g. schizophrenia, Alzheimer's disease) when compared to the measures obtained in the age- and sex-matched healthy subjects. In this regard, the TMS induced MEP modulation has been associated with the activation and interaction between specific inhibitory and excitatory mechanisms at the cortical level, which provides insight on the specific cortical processes that may underlie the neuropsychopathology of such disorders. However, evidence for the cortical origin of MEP modulation is relatively scarce and has often been collected from rather invasive procedures such as epidural recordings in patients with implanted electrodes and by demonstrating that MEP modulation is not due to the refractoriness of the corticospinal tract.

In recent years, it has become possible to combine TMS-EMG with simultaneous electroencephalography (EEG) recordings. The combination of TMS with EEG, however, has not been easy. Simultaneous EEG recording during TMS stimulation was previously unattainable because of the technological shortcomings of EEG amplifiers that would saturate for a long duration due to the large artifact produced by the magnetic stimulation. For example, application of a single TMS pulse would result in artifact lasting for several seconds after the pulse. Such long lasting artifact blocked the window of time during which neurophysiological processes such as cortical inhibition occur. Through advances in EEG amplifier technology, researchers have conducted series of studies to examine TMS paradigms in the motor cortex through simultaneous EEG and EMG recordings and in nonmotor regions of the cortex through EEG recordings.

The integration of EEG recordings with TMS-EMG will generate important neurophysiological leads in both the healthy and diseased states. First, it allows for noninvasive examination of the origin and the mechanisms through which MEPs are modulated. For example, using the TMS-EEG technology we have recently examined the EEG substrate of the MEP modulation induced by the paired pulse paradigm long interval cortical inhibition (LICI). In LICI, a conditioning stimulus precedes the test stimulus by 100 ms and suppresses the amplitude of the MEP response to the test stimulus by about $50 \%$ (Daskalakis et al., 2002a; Valls-Sole et al., 1992), a processes which is thought to be mediated by the activity of cortical GABAergic inhibitory interneurons and more specifically the activation of $\mathrm{GABA}_{B}$ receptors (McDonnell et al., 2006; Sanger et al., 2001). Through concurrently recording MEP and EEG responses, we were able to show that the LICI induced suppression of MEPs at the periphery is positively correlated with the suppression of cortical evoked potentials at the site of TMS stimulation, therefore, providing further evidence for the cortical origin of the MEP modulation in the LICI paradigm (Daskalakis et al., 2008).

A second advantage of online EEG recording is the possibility to evaluate the effects of electromagnetic induction on cortical oscillatory activity in order to identify the cortical oscillations that more closely underlie the TMS induced MEP generation and modulation. Indeed, we have recently demonstrated that following LICI application to the motor cortex, cortical oscillations in the frequency range of $1 \mathrm{~Hz}$ to $30 \mathrm{~Hz}$ are significantly suppressed, while higher frequencies (e.g. gamma oscillations $(30-50 \mathrm{~Hz})$ ) are not affected (Farzan et al., 2009). Identifying the cortical oscillatory activities that underlie TMS induced MEP modulation may provide further insight into the neurophysiology of the human motor cortex and would be valuable in improving the diagnostic and therapeutic strategies in several neuropsychiatric disorders.

Furthermore, a major advantage of combined TMS-EMG and EEG is the possibility to evaluate the cortico-cortical connectivity between motor cortices. Functional connectivity 
between cortical regions (e.g. left and right motor cortices) can be more easily probed through measuring the propagation of TMS induced cortical responses across cortical mantles, as opposed to evaluating the modulation of the peripheral activity. In this regard, we have recently demonstrated that by monitoring the propagation of TMS induced cortical evoked potentials from the site of stimulation to the contralateral motor area, it may be possible to evaluate the extent of cross talk between the left and right motor areas in healthy subjects at rest (Voineskos et al., 2010). The propagation of TMS induced cortical activity can be also investigated during various motor tasks. Such cortical indices would be of great value in several clinical populations, first for understanding the underlying pathology, for example by comparing the topographical map of signal propagation in stroke patients compared to healthy subjects, and second for optimizing more individually tailored treatment approaches.

One of the most important features of the TMS-EEG combination, however, is the ability to study the neurophysiology of non-motor regions of the cortex where a peripheral response may not be available. For example, examining the modulatory effect of LICI in the prefrontal cortex would enhance our understanding of the inhibitory mechanisms in a cortical area that is more closely associated with the pathophysiology of psychiatric disorders such as schizophrenia and depression. Indeed, using TMS-EEG technology, we have been able to demonstrate selective differences between the modulations of gamma oscillations in the dorsolateral prefrontal (DLPFC) of patients with schizophrenia in comparison to both healthy subjects and patients with bipolar disorder (Farzan et al., 2010a). This is while no deficits were observed in the MEP modulation or modulation of the cortical oscillations in the motor cortex. Therefore, TMS-EEG-EMG combination offers the possibility to monitor subtle changes in the non-motor regions of the cortex which previously were not available through examinations of the peripheral responses.

In this chapter, we provide a brief overview of the past, present and the future status of the TMS-EMG-EEG research. First, we provide a description of the working principle of TMS. We proceed by introducing commonly used TMS-EMG paradigms while providing evidence for the cortical mechanisms that are thought to be involved in the generation and modulation of the TMS evoked MEPs in each TMS paradigm. Then, the way in which TMSEMG and EEG can be integrated are described along with examples of recent findings which illustrate the validity, reliability and thus the power of combined measurements. Finally, we highlight the application of these techniques in healthy and diseased states, and conclude by briefly discussing the future directions for the TMS-EMG-EEG research.

\section{Examining motor pathways through cortical stimulation}

\subsection{In vitro and animal studies}

In vivo examination of the mammalian motor pathways can be traced back to electrical stimulation of the motor cortex of cats under anesthesia (Adrian \& Moruzzi, 1939). In these studies, electric shocks were delivered to an exposed area of the cat motor cortex and the potential changes in the pyramidal tracts were recorded through thin wire electrodes inserted into the dorsal surface of the medulla (Adrian \& Moruzzi, 1939). Such direct recordings from the pyramidal tracts demonstrated that the stimulation of the motor area results in an excitatory response followed by a period of complete inactivity of the pyramidal tract (Adrian \& Moruzzi, 1939). In subsequent experiments, it was further shown that the excitatory response lasted up to $20 \mathrm{~ms}$ which was succeeded by a silent period that 
would last about 200 ms (Krnjevic et al., 1964). It was also shown that the repetitive application of electrical stimulation with a frequency of 5 to 10 pulses per second (i.e. 5-10 $\mathrm{Hz}$ ) would prolong the duration of the inhibitory period (Adrian \& Moruzzi, 1939), while at higher stimulation frequencies (e.g. 20-50 Hz) convulsive firing would be generated followed by even a longer period of inhibition. This long lasting inhibitory phase, which was unlikely to be a result of the refractory period that lasts only about $4 \mathrm{~ms}$, was associated with activation of cortical GABAergic inhibitory interneurons (Krnjevic et al., 1964). Similarly, later in vitro stimulation of human neocortical slices demonstrated similar findings (McCormick, 1989). In this regard, the intracellular recordings from the human temporal lobe slices showed three distinct response phases: an initial fast excitatory post synaptic potential (EPSP) followed by a fast inhibitory post synaptic potential (IPSP) and finally a slow IPSP (McCormick, 1989). It has been suggested that glutamate may underlie the initial EPSP and pharmacological assessments have associated the fast inhibitory response with activation of $\mathrm{GABA}_{\mathrm{A}}$ receptors and the second slow acting response with activation of $\mathrm{GABA}_{\mathrm{B}}$ receptors (McCormick, 1989).

\subsection{Cortical stimulation in humans}

Transcranial electrical stimulation (TES) can be regarded as the first technique that permitted the focal stimulation of the human motor cortex by the placement of external electrodes on the scalp (Merton \& Morton, 1980). In TES, the electrical stimulation is delivered through a set of two electrodes, one positioned over the motor cortex, and the other $4 \mathrm{~cm}$ anterior to the first electrode. Merton and Morton demonstrated that TES applied to the motor cortex through these electrodes could generate a MEP at the periphery that could be captured through EMG recordings. It has been suggested that the activation of cortex through TES is similar to the direct electrical stimulation of the exposed motor cortex of non-human primates (for a review see (Day et al., 1989)). However, TES is a painful procedure, owing to the dissipation of a significant amount of current over the skin before it reaches the brain tissue. For example, to evoke a peripheral response a capacitor that is charged up to $2000 \mathrm{~V}$ has to be discharged within a very short time of about $10 \mu \mathrm{s}$. As such, TES is mostly used to examine motor pathways in patients with spinal cord injuries and it is often administered during anesthesia. Since the introduction of TMS in 1985, it has now become possible to non-invasively examine the excitability of the motor pathways in awake healthy humans.

\subsubsection{Working principles of TMS}

Activation of neurons through TMS technique is based on the Faraday's law of induction of electric current via a time-varying magnetic field (Hallett, 2000). In TMS, a magnetic coil is placed tangential to the subject's scalp over the motor area, and intense time-varying current pulses of about $8 \mathrm{kA}$ run though the coil. The time-varying current in the coil induces a time-varying magnetic field perpendicular to the plane of the coil, and hence the surface of brain tissue, which in turn induces an electric field parallel to the plane of the coil in the brain tissue. The duration of a single pulse TMS is typically within a range of 200 to $600 \mu \mathrm{s}$. Unlike TES, the induced magnetic field passes through skin, scalp and skull unimpeded, and as such, TMS is less painful than TES. The maximum magnetic field induced by a magnetic stimulator is about 2.5 Tesla which can induce electric field and tissue current of magnitude 500 volt $/ \mathrm{m}$ and $15 \mathrm{~mA} / \mathrm{cm}^{2}$, respectively. Furthermore, the strength of the 
induced magnetic field falls exponentially with increasing distance from the surface. Thus, TMS activates the brain tissue in the outer most $2-2.5 \mathrm{~cm}$.

Due to the horizontal orientation of TMS induced current in the cortex, TMS activates horizontally-oriented interneurons with a higher probability compared to the verticallyoriented pyramidal neurons. Therefore, focal TMS activates pyramidal neurons transynaptically, rather than at the axon of pyramidal tract neurons (Rothwell, 1997). For example, when the handle of the magnetic coil is pointed backward, approximately $45^{\circ}$ to the mid-sagittal line, the induced current in the brain is perpendicular to the presumed direction of the central sulcus (Day et al., 1989). The indirect activation of pyramidal neurons by TMS is supported by epidural recordings of descending volleys following application of TMS and TES in patients who have spinal stimulators implanted in the epidural space. The results of epidural recordings have demonstrated that TES produces an early descending volley (referred to as direct wave or D-wave) which at a high intensity of stimulation is followed by indirect multiple waves (I-waves) that peak with a delay period of $1.5 \mathrm{~ms}$ from one another. By contrast, focal monophasic TMS results primarily in I-waves, and the number of these I-waves and their amplitude increase with the intensity of stimulation. It is reported that TMS may also generate a D-wave at very high intensities (Di Lazzaro et al., 1999b). D-wave and I-waves were originally observed following electrical stimulation of pyramidal tracts of cats. I-waves have been associated with the synaptic activation of pyramidal neurons through excitatory interneurons while D-waves are associated with a direct activation at the axon of pyramidal neurons (reviewed in (Ziemann \& Rothwell, 2000)).

\subsubsection{Factors affecting the TMS induced current}

Several factors affect the depth and pattern of tissue activation and shape of the TMS induced MEP at the periphery. The geometry of the coil (e.g. circular, figure-8, doublecone, H-coil), the shape of the magnetic pulse generated by the coil (e.g. monophasic or biphasic pulse), and the type of material used to construct the core (e.g. solid-core or aircore) affect the characteristics of the induced current. In regards to the coil geometry, a figure-of- 8 coil has a greater focality than a circular coil while the newer generations of deep coils, such as H-coil, have the greatest depth of stimulation. The shape of the induced current also varies between each coil type. For example, the induced electrical field is maximal under the rim of the circular coil, and the strength reduces in the centre, while in the figure-eight coil, the maximum field is in the centre of the coil at the junction of the two circular wings. In addition, biphasic TMS pulses are suggested to be more powerful than monophasic pulses and they generate a more complex pattern of neural activation (Di Lazzaro et al., 2001). Finally, solid-core coil are more efficient than air-core coils in transferring the electrical energy to magnetic field and as a result dissipate less heat. In addition to these parameters, the intensity of the pulse and the positioning of the coil affect the induced current and consequently the activation pattern. Consequently, by changing the intensity and the orientation of the coil different populations of neurons can be activated.

\section{TMS combined with electromyography}

TMS has been used for both therapeutic and diagnostic purposes (Rossini \& Rossi, 2007). In relation to its diagnostic application, TMS-EMG provides a tool to assess the timing of 
cortical processes, cortico-cortical connectivity, cortical inhibition and facilitation, and the interaction between cortical processes (Anand \& Hotson, 2002; Chen, 2004; Daskalakis et al., 2004; Di Lazzaro et al., 2004; Pascual-Leone et al., 2000; Sanger et al., 2001). In this section, several of these TMS paradigms will be discussed in more details, and wherever applicable, evidence supporting their neurophysiological mechanisms will be provided.

\subsection{Motor threshold}

Motor threshold is often determined by applying single pulse TMS to the motor cortex while the coil is placed at the optimal position for eliciting MEPs from a target muscle. The two muscles which are more easily accessible by TMS stimulation are the abductor pollicis brevis (APB) and the first dorsal interosseous (FDI) muscles. Resting motor threshold (RMT) for a target muscle can be determined through applying single pulse TMS to an area of the motor cortex that generates the largest MEP amplitude for the target muscle, and it is defined as the minimum stimulus intensity that can elicit an MEP of more than $50 \mu \mathrm{V}$ in at least five out of ten trials in the target muscle (Rossini et al., 1994). It has been demonstrated that NMDA antagonists such as ketamine reduce motor threshold, the block of voltagegated sodium channels increases motor threshold, and GABA has no influence on motor threshold (for a review refer to (Ziemann, 2004)).

\subsection{Short interval cortical inhibition}

In short interval cortical inhibition (SICI), a subthreshold conditioning stimulus delivered 1 to $5 \mathrm{~ms}$ prior to a suprathreshold test stimulus suppresses the MEP response to the test stimulus compared to the MEP response to a test stimulus delivered alone (Kujirai et al., 1993). It has been suggested that suppression of MEP occurs at the cortical level and is not due to the refractoriness of corticospinal tract (Di Lazzaro et al., 1998; Kujirai et al., 1993). For example, epidural recordings have shown that a preceding subthreshold stimulus that does not produce an I-wave by itself, suppresses the late I-waves of a suprathreshold stimulus that is delivered after 1 to $5 \mathrm{~ms}$ (Di Lazzaro et al., 1998). In addition, it was shown that magnetic conditioning stimulus did not suppress the response to an electrical test stimulus which activates the axon of the corticospinal tract (Kujirai et al., 1993). Finally, an electrical conditioning stimulus was less effective in suppressing the response to a magnetic test stimulus suggesting that the inhibition observed in SICI is not due to the refractoriness of corticospinal tract (Kujirai et al., 1993). SICI has been associated with the activity of $\mathrm{GABA}_{\mathrm{A}}$ receptor mediated inhibitory neurotransmission, as $\mathrm{GABA}_{\mathrm{A}}$ agonists such as lorazepam have been shown to enhance SICI as well as the suppression of the late I-waves (Di Lazzaro et al., 2000).

\subsection{Intracortical facilitation}

In this paradigm, a subthreshold conditioning stimulus delivered 7 to $20 \mathrm{~ms}$ prior to a test stimulus, facilitates the MEP response to the test stimulus (Kujirai et al., 1993). It has been suggested that intracortical facilitation (ICF) is an index of the excitability of the excitatory circuits in motor cortex (Ziemann et al., 1996). Pharmacological studies by Ziemann et al. demonstrated that some NMDA antagonists (such as dextrometorphan) and $\mathrm{GABA}_{\mathrm{A}}$ agonists (such as lorazepam) reduce ICF, while GABA $_{B}$ agonists (such as baclofen) increase ICF (Ziemann, 2004). The reduction of ICF by NMDA antagonist suggests that glutamate may play a role in mediating the cortical response observed in ICF. 


\subsection{Long interval cortical inhibition}

In long interval cortical inhibition (LICI), a suprathreshold conditioning stimulus, delivered within 50 to $200 \mathrm{~ms}$ prior to a suprathreshold test stimulus, suppresses the response to the test stimulus compared to the response to the test stimulus delivered alone (Valls-Sole et al., 1992). Epidural recordings suggest that this suppression has a cortical origin at ISI of 100 to 200 ms (Chen et al., 1999; Di Lazzaro et al., 2002a; Nakamura et al., 1997). For example, it was shown that LICI at ISI of 100, 150 and 200 ms resulted in suppression of descending volleys, in particular the late I-waves (Chen et al., 1999; Di Lazzaro et al., 2002a; Nakamura et al., 1997). Several lines of evidence suggest that the LICI induced suppression of MEP responses is related to the activity of $\mathrm{GABA}_{B}$ receptor mediated inhibitory neurotransmission ((McDonnell et al., 2006; Sanger et al., 2001); also reviewed in (Kapogiannis \& Wassermann, 2008)). It has been suggested that in LICI, activation of the $\mathrm{GABA}_{\mathrm{B}}$ receptors by the conditioning stimulus, inhibits excitation of the motor cortex to the test stimulus that would have otherwise excited the cortex if delivered alone. The $\mathrm{GABA}_{\mathrm{B}}$ receptor activation is suggested to peak around 150 to $200 \mathrm{~ms}$ post stimulus (McCormick, 1989), and interestingly LICI is optimal when conditioning stimulus precedes the test stimulus by 100 to $150 \mathrm{~ms}$ (Sanger et al., 2001).

\subsection{Cortical silent period}

Cortical silent period (CSP) involves a transient suppression of EMG activity following delivery of a single suprathreshold TMS pulse to the motor cortex during voluntary contraction of the target muscle. Previous studies suggest that early part (i.e. first $50 \mathrm{~ms}$ ) of CSP is due to spinal mechanism, while the later part is mediated by cortical inhibition (Chen et al., 1999; Inghilleri et al., 1993). Similar to LICI, CSP is also associated with $\mathrm{GABA}_{B}$ receptor mediated inhibitory neurotransmission (Chen et al., 1999; Nakamura et al., 1997; Siebner et al., 1998; Werhahn et al., 1999). For example, Siebner et al. reported a significant prolongation of the CSP duration following a continuous intrathecal administration of baclofen in a patient with generalized dystonia (Siebner et al., 1998). Also, tiagabine, a GABA uptake inhibitor, was shown to enhance LICI and prolong CSP duration (Werhahn et al., 1999). Furthermore, administration of vigabatrin, a selective GABAergic drug that increases the availability of GABA in the brain, has also been shown to enhance LICI and CSP (Pierantozzi et al., 2004). In addition, we have recently shown a significant positive relationship between the suppression of MEP amplitudes in LICI (with an ISI of $100 \mathrm{~ms}$ ), and the duration of the silent period in the CSP paradigm in healthy subjects (Farzan et al., 2010b).

\subsection{Interhemispheric inhibition}

Interhemispheric inhibition (IHI), also known as transcallosal inhibition, refers to a phenomenon in which a suprathreshold TMS pulse delivered to one hemisphere can inhibit the MEP response to a suprathreshold TMS pulse that is delivered within 6 to $50 \mathrm{~ms}$ to the opposite hemisphere (Ferbert et al., 1992; Gerloff et al., 1998). IHI is conventionally measured at an ISI of 6 to $12 \mathrm{~ms}$, however MEP modulation at an ISI of $40 \mathrm{~ms}$ has also been demonstrated but suggested to be mediated by different mechanisms (Chen et al., 2003; Ni et al., 2009). By demonstrating the suppression of the late I-waves, epidural recordings have confirmed that IHI is mediated cortically and most likely through transcallosal pathways (Di Lazzaro et al., 1999a). Furthermore, it was shown that the magnetic conditioning stimulation 
of contralateral hemisphere did not significantly inhibit the response to an electrical test stimulus (Ferbert et al., 1992). Moreover, triple pulse TMS studies have demonstrated that IHI modulates the MEP response to SICI and LICI which, as previously described, are cortically mediated phenomenon, providing further evidence that IHI itself has a cortical origin (Daskalakis et al., 2002b). Moreover, recently a positive association was found between IHI and the integrity of callosal motor fibers (Wahl et al., 2007). The integrity of callosal motor fiber was examined through diffusion tensor imaging and by measuring the fractional anisotropy, a measure of white matter tract integrity. The results of this study suggest that TMS-EMG combination may also provide useful information about the integrity of the callosal motor fibers.

\subsection{Sensory afferent inhibition}

Sensory afferent inhibition (SAI) involves the modulation of motor cortex excitability by means of an afferent input produced by the electrical stimulation of digital or medial nerve at the wrist (Tokimura et al., 2000). In SAI, a preceding electrical stimulation of the peripheral nerve suppresses the MEP amplitude elicited by the application of a single pulse TMS to the contralateral motor cortex. There are two forms of SAIs that are suggested to be mediated by slightly different mechanisms: short latency SAI, and long latency SAI. Short SAI, which is investigated more thoroughly, occurs when the peripheral stimulation is delivered about $25 \mathrm{~ms}$ prior to the TMS pulse, while long SAI is observed when the sensory input is delivered about $200 \mathrm{~ms}$ prior to the TMS pulse. Comparison of TMS and TES and recordings of descending volleys suggest that SAI occurs at the cortical level (Tokimura et al., 2000). The molecular underpinnings of SAI are complex and SAI likely relates to an interaction between several neurotransmitter systems with the possible involvement of the cholinergic neurotransmission (Di Lazzaro et al., 2005; Di Lazzaro et al., 2007; Di Lazzaro et al., 1999c).

\subsection{Repetitive TMS}

It has been demonstrated that repetitive delivery of TMS pulses (rTMS) as well as repetitive application of TMS pulses paired with electrical peripheral nerve stimulation results in an MEP modulation which would outlast the duration of TMS delivery. Conventionally, rTMS is administered through application of TMS pulses at a frequency of $0.5 \mathrm{~Hz}$ (ISI of $2 \mathrm{~s}$ ) to 50 $\mathrm{Hz}$ (ISI of $20 \mathrm{~ms}$ ). It is thought that the repetitive administration of TMS pulses results in summation of TMS induced alteration of cortical activity, thereby causing an effect which may outlast the stimulation period (Chen et al., 1997; Pascual-Leone et al., 1994). The alteration of cortical activity by rTMS has been typically indexed as a suppression or potentiation of MEP responses to the standard single pulse TMS paradigm before and after an active rTMS session or sometimes by comparing the after effects of active versus sham rTMS. A few studies have also evaluated the effect of rTMS on intracortical inhibition by measuring the changes in CSP and MEP modulation by paired pulse TMS before and after rTMS administration (Daskalakis et al., 2006; Fitzgerald et al., 2004). The results of these studies have demonstrated that on average low frequency rTMS $(<5 \mathrm{~Hz})$ applied to the motor cortex suppresses the MEP amplitudes for about $30 \mathrm{~min}$ following the end of the rTMS administration ((Chen et al., 1997); reviewed in (Fitzgerald et al., 2006)). The effect of fast frequency rTMS (> $5 \mathrm{~Hz}$ ) has been shown to be the facilitation of the MEP amplitudes, however results have been somewhat inconsistent across laboratories (Daskalakis et al., 
2006; Fitzgerald et al., 2006; Maeda et al., 2000), perhaps suggestive of a biphasic effect of fast rTMS with a short period of increased excitability followed by a phase of increased inhibition.

It has been suggested that rTMS combined with EMG may be an effective tool for probing and modifying cortical plasticity. Neural plasticity, a mechanism thought to be critical for learning and memory, represents synaptic strength and the degree of signal transmission between coactive cells which can be weakened (long term depression, LTD) or strengthened (long term potentiation, LTP) in response to the presentation of a strong or weak synchronous synaptic stimulation. The lasting effect of rTMS, reflected through suppression and facilitation of TMS induced MEPs, has been suggestive of the potential value of TMS in probing and inducing LTP and LTD like plasticity in the cortex (reviewed in (Pell et al., 2010)). Owing to this lasting effect, application of rTMS to different brain regions may cause behavioural changes that would outlast the duration of rTMS stimulation (For a review refer to (Ridding \& Rothwell, 2007)). This aspect of rTMS has been used to improve and restore functional impairments in several disorders such as stroke, movement disorders, addiction, cognitive as well as psychiatric disorders, with the most promising outcomes observed in the treatment of depression (for a review see (Slotema et al., 2010; Wassermann \& Lisanby, 2001)).

Plasticity may also be induced through repeated presentation of a pair of somatosensory afferent stimulation and cortical simulation. This can be achieved through the TMS paradigm paired associative stimulation (PAS) which involves pairing of the peripheral electrical nerve stimulation and single pulse TMS of the contralateral motor cortex repeated at a frequency of $0.1 \mathrm{~Hz}$ (Classen et al., 2004; Stefan et al., 2002; Stefan et al., 2000). Choosing an appropriate ISI between peripheral and cortical stimulation (e.g. $25 \mathrm{~ms}$ ), PAS has been shown to induce a potentiation of MEPs in the target muscle which would last about $40 \mathrm{~min}$ following the end of the stimulation (Tsuji \& Rothwell, 2002). Moreover, pharmacological studies showed that NMDA antagonists suppressed the PAS induced MEP facilitation, while $\mathrm{GABA}_{\mathrm{A}}$ receptor antagonist had no effect (Stefan et al., 2002). Some experimental findings suggest that PAS induced effects have a cortical origin. First, following the PAS intervention and in the presence of facilitated MEPs, the F waves recorded from the median nerve stimulation reflected no change in the excitability of $\alpha$-motor neurons of the median nerve (Stefan et al., 2002). Second, the MEPs evoked by the electrical stimulation of the brainstem remained unchanged following PAS intervention, while the MEP responses to single pulse TMS were increased compared to the baseline MEP responses (Stefan et al., 2000). These findings along with the transynaptic activation of pyramidal neurons by TMS suggest that PAS-induced MEP potentiation is likely mediated through transynaptic mechanisms in the motor cortex (Stefan et al., 2000), seemingly related to the associative (i.e. Hebbian) LTP of synaptic efficacy (Stefan et al., 2002). According to animal studies and in vitro recordings from neocortical slices, associative LTP occurs at an input to a postsynaptic cell which is conditioned by a synchronous activation of another input to that same cell (Buonomano \& Merzenich, 1998). Given that PAS induced MEP potentiation is ISIdependant, it is inferred that the PAS induced plasticity is likely due to the synchronous arrival of the somatosensory afferent input and the TMS induced activation of horizontally oriented interneurons in the sensorimotor cortex (Classen et al., 2004; Stefan et al., 2002; Stefan et al., 2000).

Recently, it was shown that the delivery of repetitive TMS through a protocol named theta burst stimulation (TBS) may be a more efficient way of altering cortical excitability and 
probing LTP and LTD like cortical responses (Huang et al., 2005). Theta burst stimulation involves application of 3 bursts of $50 \mathrm{~Hz}$ rTMS repeated every $200 \mathrm{~ms}$ either continuously for a total of $40 \mathrm{~s}$, or intermittently (every $8 \mathrm{~s}$ ) for a total of $3 \mathrm{~min}$. The continuous (cTBS) and intermittent delivery of TBS (iTBS) have been shown to result in suppression and facilitation of cortical excitability, respectively, as indexed through suppression and facilitation of MEPs at the periphery (Huang et al., 2005). More importantly, it has been shown that despite the relatively short duration of TBS administration (40 s in cTBS and $3 \mathrm{~min}$ in iTBS) compared to the conventional rTMS ( $25 \mathrm{~min})$, the alteration of cortical excitability by TBS can last for about $70 \mathrm{~min}$ which is more than twice as long as the duration of the after effects reported in the conventional rTMS approaches (Di Lazzaro et al., 2011; Thut \& Pascual-Leone, 2010).

While the combination of TMS with EMG has been instrumental in assessment of several cortical processes, measuring the effect of cortical stimulation at the periphery has several limitations. First, and foremost and as the motor cortex is concerned, the exact mechanism underlying the generation and modulation of the TMS evoked MEPs remains unclear. While TMS-EMG studies in combination with epidural recordings and electrical stimulation can to some extent confirm the cortical origin of MEP responses, such recordings are invasive and only possible in specific subgroups of patients suffering from neurological disorders. To examine the cortical mechanisms involved in the generation of MEPs in healthy human subjects, therefore, a reliable non-invasive recording from the intact brain is desirable. In the next two sections, we will address how a direct but non-invasive recording of cortical activity would complement the conventional TMS-EMG measures.

\section{Recording cortical activity in humans through electroencephalography}

Recording electrical activity from the intact surface of mammalian cortex dates back to recordings from the dog's skull in 1870s and to the works of Richard Caton and Vladimir Pravdich-Neminski. Later in 1920s, Hans Berger recorded brain waves from the surface of human scalp and named the technique electroencephalography (EEG) (Buzsáki, 2006; Swartz \& Goldensohn, 1998). To date, EEG is still the cheapest and the most widely used neurophysiological technique for measuring cortical local field potentials (Buzsáki, 2006). In EEG, electrical activity of the cerebral cortex is monitored by placing multiple electrodes on the scalp. While TMS induced MEPs, for example, are an outcome of an action potential generated by the activation of pyramidal neurons, cortical potentials recorded from the scalp are not a measure of action potential but they represent the activity of slow and fact acting EPSPs and IPSPs mostly originating from the surface of the cortex (Buzsáki, 2006). Cortical potentials recorded through EEG, therefore, represent the oscillatory activity of the underlying brain tissue. Comparison between monozygotic and dizygotic twins has revealed a high heritability for the temporal structure of amplitude fluctuations in EEG oscillations, implying that EEG may be a reliable neurophysiological technique (LinkenkaerHansen et al., 2007).

\subsection{Generation and function role of cortical oscillations}

While the typical firing rate of a motor unit does not exceed the frequency range of $6-35 \mathrm{~Hz}$, neuronal tissues are capable of oscillating within a wide frequency band ranging from less than $1 \mathrm{~Hz}$ to as high as $600 \mathrm{~Hz}$. The results of in vivo and in vitro recordings suggest that the brain generates finite number of discrete frequency bands which are conventionally 
categorized as delta $(1-4 \mathrm{~Hz})$, theta $(4-7 \mathrm{~Hz})$, alpha $(8-12 \mathrm{~Hz})$, beta $(12-28 \mathrm{~Hz})$ and gamma $(30-50 \mathrm{~Hz})$ oscillations (Buzsáki, 2006), with some recent experimental findings suggestive of the presence of slower $(0.02 \mathrm{~Hz}$ to $1 \mathrm{~Hz}$ ) and faster oscillations (up to $600 \mathrm{~Hz}$ ) (Buzsáki, 2006). Several factors can affect the frequency of cortical oscillations in humans. In particular, the amplitude and frequency of cortical oscillations have been shown to vary between the mental states of sleep, resting, wakefulness, movement or engagement in complex cognitive functioning. Furthermore, it has been shown that each cortical region may have a specific frequency, with slower alpha oscillations observed more prominently over the posterior and parietal lobes and higher gamma frequencies predominant in the anterior region of the brain (i.e. prefrontal cortex)(Rosanova et al., 2009).

The exact mechanisms underlying the generation of cortical oscillations are not yet fully understood. It is suggested that the presence of various channels in the cell membrane provides neurons with intrinsic properties that allow them to oscillate at various frequencies (Buzsáki, 2006). There are several channels in the cell membrane of neurons through which ions move in and out of the cell. The state of these channels (i.e. open versus close state) is regulated by several mechanisms which are collectively referred to as gating. Four gating mechanisms have been identified: voltage gating, ligand gating, ion-dependent gating, and second-messenger gating. It is suggested that a change in the membrane voltage, release of neurotransmitters, or a change in the concentration of ions can change the state of membrane channels and give rise to an oscillatory activity (Buzsáki, 2006). It is further demonstrated that different population of neurons may selectively respond to inputs of different frequencies (Hutcheon \& Yarom, 2000; Pike et al., 2000). For example, in hippocampus of rats, fast spiking GABAergic interneurons respond with highest temporal precision to frequencies in the gamma band, whereas pyramidal cells respond to lower frequency rhythmic inputs (Pike et al., 2000). Furthermore, it has been demonstrated that there may be an inverse relation between the size of the neuronal pool and the frequency of oscillations. That is, low frequency oscillations are generated by a larger population of neurons while higher frequency oscillations may be generated by a smaller number of neurons (Buzsáki, 2006).

The functional role of various cortical oscillations is currently under investigation. The functional role of slow frequency delta oscillations, which in healthy subjects are typically observed during deep sleep, is associated with learning and motivational processes (Knyazev, 2007). The cortical activity within the theta-band has been linked to memory functions, and emotional regulations (Knyazev, 2007). Alpha-band oscillations which are predominant during wakeful resting state are suggested to arise from cortical operations in the absence of sensory inputs, and also may reflect the disengagement of task-irrelevant brain areas (Palva \& Palva, 2007). Cortical oscillations within the beta range are shown to be more dominant in the central region of the human cortex, suggestive of their functional role in movement execution and control. It has recently been hypothesized that the functional role of beta oscillations may be related to the maintenance of sensorimotor and cognitive state. That is, oscillations within beta band may be a signal for maintenance of status quo (Engel \& Fries, 2010). Consistent with this view, it has been suggested that prolongation of beta oscillations may result in deterioration of flexible behaviour (Engel \& Fries, 2010). Finally, the functional role of gamma oscillations has been more closely associated with cognitive control. In this regard, results of several EEG studies have found an association between the oscillataory acitivity in the gamma range and perceptual binding such as the construction of object representations, basic information processing such as sensory 
processing, and complex cognitive processes such as working memory (Buzsáki, 2006; Engel \& Singer, 2001; Fries et al., 2007; Howard et al., 2003; Tallon-Baudry \& Bertrand, 1999; Tallon-Baudry et al., 1996; Tallon-Baudry et al., 1998).

Recordings of brain activity through EEG suggest that cortical potentials are a result of summation of different oscillatory activities. Several theories and experimental findings exist to explain why different oscillations may be active at the same time. It has been hypothesized that presence of various oscillations may provide the brain with the ability to function through multiple time scales (Buzsáki, 2006). For example, it has been proposed that different frequencies (e.g. slow versus fast oscillations) may favor different types of computation or different level of connectivity. Based on experimental findings, it was hypothesized that middle-frequency oscillations, such as theta and alpha, may represent top-down processes, while high frequency oscillations, such as gamma, may be related to bottom-up processes (von Stein et al., 2000). Furthermore, the interaction between frequency bands has been proposed to be an essential mechanism for optimal information processing (Roopun et al., 2008). One form of frequency-band interaction is frequency concatenation (Roopun et al., 2008). In this regard, two local networks that are co-activated and oscillate at two different frequency bands may interact and generate a third frequency of oscillations. The period of the new oscillation is the concatenation sum of the original two (Roopun et al., 2008). Another form of frequency-band interaction is nesting. In nesting, phase of a lower frequency band (e.g. theta) may modulate the amplitude of a higher frequency band (e.g. gamma). The theta modulation of gamma oscillations has been observed in the hippocampus ((Bragin et al., 1995); reviewed in (Roopun et al., 2008)). The oscillatory modulation of neuronal responses is suggested to play a key role in information processing perhaps by providing a temporal frame with respect to which neurons may communicate with each other (Fries et al., 2007).

\subsection{Cortical oscillations in the motor cortex}

The presence of beta oscillations over the precentral region was first reported by Jasper and Andrews EEG recordings from healthy human subjects in 1938 (Jasper and Andrews 1938). It is believed that beta frequency can be further subdivided into narrower frequency bands of low- (12-15 Hz), mid- (15-18 Hz) and high-range $(18-30 \mathrm{~Hz})$ beta oscillations, each subserving a distinct functional mechanism. A number of previous studies have attempted to explore the cortical correlate of the EMG signals in order to examine the connectivity between the peripheral muscle and the homologous motor area. This has been typically achieved through computing the coherence between the cortical oscillations measured from the motor cortex through EEG or magnetoencephalography and the EMG recordings from the corresponding peripheral muscle often obtained during voluntary isometric hand contraction (for a review see (Mima \& Hallett, 1999)). The findings of these corticoperipheral coherence studies suggest that beta oscillatory activity in the contralateral motor cortex may underlie the generation of EMG signals in the corresponding body part (Brown et al., 1998). For example, tonic hand muscle contraction has been shown to induce beta oscillations $(\sim 15-30 \mathrm{~Hz})$ in the contralateral hemisphere which are coherent with the EMG recordings at the periphery (Brown et al., 1998; Salenius et al., 1997).

The modulation of cortical oscillatory activity before, during and after motor task execution, imagination and observation has been well documented. Several studies have reported on desynchronization of alpha and beta oscillations prior to self-paced hand or finger 
movement often measured through C3 EEG electrode in the left hemisphere and C4 in the right hemisphere (Pfurtscheller et al., 2000). Following self-paced hand movement, desynchronization of alpha oscillations are observed over the contralateral motor cortex (Pineda, 2005) while beta oscillations ( 14-32 Hz) are induced shortly (within one second) after the movement termination (Pfurtscheller et al., 2000). Observation and imagination of motor movement also result in suppression of alpha oscillations in motor areas corresponding to that body part (Pfurtscheller et al., 2000). Furthermore, the peak frequency of the induced beta oscillations may differ between motor areas associated with different body parts. For example, hand movement was shown to induce an oscillatory activity within a frequency range of $16-22 \mathrm{~Hz}$ over the hand area, while slightly higher frequency oscillatory activity $(19-26 \mathrm{~Hz})$ were recorded in the foot area following foot movement (Neuper \& Pfurtscheller, 2001).

The combination of TMS with EMG has offered the possibility to trigger and quantify the magnitude of an involuntary movement, and evoke or slow down activation of a target muscle. As previously described, this is achieved through transynaptic manipulation of corticospinal pathway through application of TMS to the motor area that optimally represents the target muscle on the periphery. However, the specific cortical oscillations that underlie these TMS induced motor responses cannot be clearly identified through only EMG recordings. In the following section, it will be demonstrated that combination of online EEG recording with TMS-EMG would permit examining the functional role of cortical oscillations in each TMS paradigm. The advancement in the EEG amplifier technology that has led to the combination of these modalities will be first briefly described.

\section{Combining TMS-EMG with concurrent EEG recording}

Simultaneous EEG recording during TMS stimulation was previously unattainable because of the technological shortcomings of EEG amplifiers that would saturate for a long duration due to the large artifact produced by the magnetic stimulation. For example, application of a single monophasic TMS pulse would result in artifact lasting for several seconds after the pulse. Such long lasting artifact blocked the window of time during which neurophysiological processes such as cortical inhibition occur (the first $250 \mathrm{~ms}$ following TMS). The newer generation of EEG systems, however, can recover from the TMS artifact within a time window of 10 to $70 \mathrm{~ms}$.

The newer generations of EEG systems offer several strategies to minimize the saturation of amplifiers following TMS. In a majority of TMS-EEG systems, the saturation of EEG amplifiers is avoided by virtue of a sample and hold circuit that pins the amplifier output to a constant level starting from a few milliseconds prior to the TMS application to a few milliseconds after the pulse (Nexstim Ltd., Helsinki, Finland). Some systems are equipped with a magnetic shielding technology in which the EEG electrodes and the amplifiers are shielded from the magnetic artifact (Advanced Neuro Technology, Enschede, Netherland). It is also possible to minimize amplifier saturation and the TMS related artifact through a combination of a wide dynamic range (e.g. recording potentials ranging from -200 to 200 $\mathrm{mV}$ in amplitude and with a resolution much lower than $1 \mu \mathrm{V})$, high frequency sampling rate (e.g. $20 \mathrm{KHz}$ ) and DC filtering (NeuroScan, Compumedics, USA). By employing any of the above strategies, EEG recording can become TMS compatible. Therefore, it is possible to continuously record EEG during TMS stimulation (for a review refer to (Ilmoniemi \& Kicic, 2009)). 


\subsection{Single pulse TMS}

Ilmoniemi and colleagues were one of the first research groups that used the interleaved TMS-EEG technique to investigate the effect of TMS on cortical excitability (Ilmoniemi et al., 1997). It was demonstrated that TMS applied to the hand representation area of the human motor cortex elicited a cortical response that spread to the adjacent ipsilateral area as well as to the homologous regions in the opposite hemisphere. It was further shown that the application of TMS to the visual cortex resulted in similar pattern of signal propagation to the contralateral areas, therefore providing evidence that the cortical potentials following motor cortex stimulation were less likely to be a result of peripheral sensory activation. This original experiment resulted in a series of studies that further characterized the EEG substrate of cortical excitability, plasticity and connectivity in healthy subjects (Esser et al., 2006; Kahkonen et al., 2001; Kahkonen et al., 2003; Komssi et al., 2002; Komssi \& Kahkonen, 2006; Nikulin et al., 2003; Paus et al., 2001; Thut et al., 2003).

In a number of previous studies it was demonstrated that single pulse TMS applied to the motor cortex generated several EEG peaks within the first $300 \mathrm{~ms}$ of TMS stimulation. The EEG components that are commonly replicated across TMS-EEG studies are a negativity at $15 \mathrm{~ms}$ (N15), a positivity at $30 \mathrm{~ms}$ (P30) followed by N45, P55, N100, P180, and N280 (Komssi \& Kahkonen, 2006). Comparing these EEG peaks with the size of MEP responses may permit examining the cortical origin of the TMS induced MEPs. In this regard, it has been shown that TMS induced MEP amplitudes positively correlate with the amplitude of the TMS induced early evoked potential, measured as the peak-to-peak amplitude of the N15-P30 component (Maki \& Ilmoniemi, 2010b). In a study by the same group, the relationship between the TMS induced MEPs and the cortical oscillatory activity was explored. It was shown that in the motor cortex, the power of mid-range beta oscillatory activity (12-18 Hz) was weaker before large MEPs as compared to smaller MEPs. Furthermore, the phase of the mid-range beta oscillations recorded over the occipital cortex correlated with the MEP amplitudes (Maki \& Ilmoniemi, 2010a). Similarly, Schutter and Hortensius examined the association between cortical oscillatory activity and MEP amplitudes during isometric hand contraction. It was reported that MEP amplitudes could be modeled by the activity of theta $(4-7 \mathrm{~Hz})$ and beta $(13-30 \mathrm{~Hz})$ cortical oscillations in the right and left primary motor cortex (Schutter \& Hortensius, 2011). These authors went a step further and applied this finding to selectively control the therapeutic outcome of transcranial alternative current stimulation (tACS) (for detailed description of tACS readers may refer to (Kanai et al., 2008)). In this experiment, they applied the tACS with two different frequency settings of theta-beta and alpha-alpha configuration which were administered on two separate days. It was shown that the theta-beta tACS significantly increased cortical excitability compared to the alpha-alpha stimulation (Schutter \& Hortensius, 2011).

A few studies have also examined the inhibitory components of TMS induced cortical potential. For example, Nikulin et al. demonstrated that following visually triggered hand movement, the N100 component of EEG response was suppressed while the MEP amplitudes were increased (Nikulin et al., 2003). This inverse relationship between the amplitudes of N100 and MEP is thought to reflect the inhibitory nature of N100. Consistent with this finding, the duration of silent period in the CSP paradigm was shown to be positively correlated with the amplitude of the N100 component (Kimiskidis et al., 2008). Interestingly, in a TMS study that investigated the effect of alcohol on TMS evoked cortical potentials, it was shown that following alcohol intake, which is thought to affect the activity 
of GABAergic neurotransmission, the amplitude of N100 response to single pulse TMS was significantly decreased (Kahkonen \& Wilenius, 2007). Finally, the effect of age, TMS intensity, sensory attention and response preparation on TMS induced N100 response was investigated in a TMS study conducted in motor cortex of healthy children within an age range of 6-10 years. It was shown that N100 response was attenuated following a task that required a fast response, providing further evidence that N100 was likely related to an inhibitory process (Bender et al., 2005). In addition, N100 response was reported to be larger in children compared to N100 responses of adults in previous studies and a negative correlation was found between N100 response and age in children, suggesting that N100 response may undergo maturation (Bender et al., 2005). Collectively, these findings suggest that GABAergic inhibitory mechanism may underlie the N100 generation. If proved a reliable measure, N100 may be used as a marker for investigating the integrity of the inhibitory responses in healthy and pathological conditions.

\subsection{Paired Pulse TMS}

\subsubsection{Short interval cortical inhibition and intracortical facilitation}

One of the first studies evaluating the modulatory effect of paired pulse TMS on cortical evoked potentials is the experiments conducted by Paus et al. (Paus et al., 2001). In this study, SICI and ICF paired pulse paradigms were applied to the left motor cortex of healthy subjects and the amplitude and frequency of EEG responses were compared between single and paired pulse conditions. In this study, three prominent EEG peaks were observed following the singe pulse TMS: N45, P55 and N100. Furthermore, it was shown that single pulse TMS resulted in synchronization of beta oscillations which lasted for several hundred milliseconds after the TMS delivery. Following the ICF paired pulse paradigm, which as discussed before is designed to examine intracortical facilitation, the presence of a subthreshold conditioning stimulus (at an ISI of $12 \mathrm{~ms}$ ) significantly reduced the amplitude of the EEG N45 component. In addition, the beta oscillatory activity that was recorded following ICF was reported to be smaller as compared to the oscillatory activity following the single pulse condition. By contrast, a conditioning stimulus delivered at an ISI of $3 \mathrm{~ms}$ (SICI paradigm) had no effect on either the N45 or beta oscillatory activity.

In a recent study, similar paired pulse experiments were conducted to further confirm and expand the above mentioned findings (Ferreri et al., 2010). In this study, SICI (ISI of $3 \mathrm{~ms}$ ) and ICF (ISI of $11 \mathrm{~ms}$ ) paradigms were applied to the left motor cortex of healthy subjects. Several EEG components were observed following single pulse TMS including: N7, P13, N18, P30, N44, P60, N100, P190 and N280. These EEG components were further identified for each stimulation condition and each EEG electrode (19 electrodes). The authors then examined the correlation between the MEP amplitudes and the amplitude of each EEG components in each electrode, after the three TMS conditions of suprathreshold single pulse and the paired pulse conditions of SICI and ICF. It was observed that depending on the stimulation condition (single pulse, SICI or ICF), MEP amplitudes correlated with different EEG components and at a different cortical region. For example, a positive correlation was observed between the MEP amplitude and the P30 component over the non-stimulated motor cortex following the single pulse condition and in the F4 (contralateral prefrontal cortex) following the paired pulse condition. Moreover, N44 peak was attenuated in the contralateral cortex more prominently in the ICF condition, a finding consistent with the earlier report of attenuation of N45 flowing ICF (ISI of $12 \mathrm{~ms}$ ) as described above (Paus et al., 2001). Furthermore, it was noted that the N100 component was strongest over the 
stimulation area (C3 electrode) and its amplitude was larger following the paired pulse conditions as compared to the single pulse condition. Collectively these findings suggest that TMS-EEG offers the possibility to examine not only the MEP-EEG relationship but also the connectivity between cortical regions as it may be evident by comparing the TMS induced EEG activity at the stimulation site with the EEG components recorded in the remaining electrodes.

\subsubsection{Long interval cortical inhibition}

As briefly described in the introduction, the cortical correlate of LICI has been recently investigated through the combination of TMS-EMG with simultaneous EEG recording. That is, we demonstrated that EMG measures of LICI, measured as the suppression of MEPs in the paired pulse condition compared to the single pulse conditions, were positively correlated with the suppression of the cortical evoked potentials at the site of stimulation (C3 electrode) in the paired pulse condition compared to the single pulse conditions (Daskalakis et al., 2008). In this study, however, instead of examining the peak-to-peak amplitude of EEG components separately, the TMS induced cortical response was identified as the area under the rectified EEG response following the single and paired pulse conditions within the time window of $50 \mathrm{~ms}$ (the earliest reliable recording without TMS artifact) to $150 \mathrm{~ms}$ (the maximum peak of $\mathrm{GABA}_{\mathrm{B}}$ receptor activation). Similarly, the potentiation of MEP response was also examined by comparing the area under the rectified MEP response between the single and paired pulse condition.

Furthermore, in a separate study we found a positive correlation between the duration of the silent period indexed through the CSP paradigm and the extent of EEG and EMG suppression following LICI in a subset of subjects who received both LICI and CSP in their left motor cortex (Farzan et al., 2010b). A key observation was that the silent period seemed to be more strongly correlated with the EEG measures of LICI as compared to the EMG measures of LICI (Farzan et al., 2010b). This observation suggests that EEG measures of LICI maybe a more precise technique for evaluating and comparing the cortical mechanisms that may be common across TMS-EMG paradigms. For instance, it has been suggested that the early $(<50 \mathrm{~ms}$ ) part of the silent period in the CSP paradigm may originate from spinal mechanism (Chen et al., 1999; Inghilleri et al., 1993). Therefore, by comparing CSP with both EEG and EMG measures of LICI (and other paired pulse paradigms), it may be possible to approximate to what degree each of these paradigms originate from the cortical rather than spinal mechanisms and also to what extent they are mediated by similar processes.

Furthermore, using the above methods, for the first time we examined the modulatory effect of LICI in the DLPFC, a non-motor region of the cortex which is implicated in a number of neurological and psychiatric illnesses (Daskalakis et al., 2008). We were able to show that similar to the motor cortex, LICI applied over the left DLPFC resulted in significant suppression of cortical evoked potentials ( $30 \%$ suppression) in the vicinity of TMS application (i.e. AF3 electrode). However, it was also shown that the extent of EEG suppression following LICI in the DLPFC was not correlated with the EEG or EMG suppression following the application of LICI to the motor cortex (Farzan et al., 2010b), a finding which may further reflect the valuable integration of EEG and paired pulse TMS which allow us to both measure and compare the neurophysiology of motor and non-motor regions of the cortex.

In line with this view, we also examined the effect of LICI on cortical oscillatory activity at the site of TMS stimulation following the LICI application in both the motor cortex and the 
DLPFC (Farzan et al., 2009). Interestingly, it was observed that LICI applied over the motor cortex had a different modulatory effect on the cortical oscillations as compared to the LICI applied to the DLPFC (Farzan et al., 2009). In the motor cortex, delta, theta and alpha oscillations were suppressed without any suppression of beta and gamma oscillations. By contrast, all cortical oscillations were significantly suppressed following the application of LICI to the left DLPFC. The differential effect of LICI on fast cortical oscillations (e.g. gamma oscillations) in the prefrontal and motor cortices provides more insight about the functional roles of these cortical oscillations in each cortical mantel.

\subsubsection{Interhemispheric inhibition}

Through TMS-EEG it is possible to examine the correlation between functional and structural interhemispheric connectivity in both motor and non-motor regions of the cortex. This is possible through application of suprathreshold single pulse TMS to one hemisphere and examining the propagation of TMS induced cortical evoked activity to the contralateral hemisphere, a cortical process which we suggest may be mediated by similar mechanisms that underlie MEP suppression observed in the conventional IHI paradigm (Voineskos et al., 2010). Using TMS-EEG and diffusion tensor imagining, we investigated the extent of interhemispheric signal propagation from the stimulated site to the contralateral hemisphere as a function of integrity of callosal fibers (Voineskos et al., 2010). We showed that following suprathreshold TMS applied to the left motor cortex, the amount of cortical evoked activity that reached the contralateral hemisphere was inversely correlated with the integrity of callosal motor fibers but not with the integrity of anterior callosal fibers, suggesting an anatomically specific structural-functional relationship. Similarly, we found an anatomically-specific relationship between the extent of interhemispheric propagation of TMS-induced cortical activity from the left DLPFC to the right DLPFC and the fractional anisotropy of anterior callosal fibers (genu) connecting left and right DLPFC, and not with the integrity of callosal motor fibers. That is, in subjects that fractional anisotropy of callosal motor fibers was larger, the propagation of suprathreshold TMS induced activity to the contralateral motor cortex was of smaller magnitude, and in subjects that fractional anisotropy of genu fibers was larger, the extent of propagation of suprathreshold TMS induced activity to the contralateral DLPFC was of a smaller value. These results collectively suggest that in the presence of suprathreshold stimulus, callosal fibers may regulate the cross talk between hemispheres perhaps through similar mechanism that underlie the inhibition of MEPs in the contralateral hand muscle in the conventional IHI paradigm.

\subsubsection{Sensory afferent inhibition}

The interleaved TMS-EEG was also used to examine the correlation between the cortical and peripheral effects of SAI. In a study conducted by Bikmullina et al., it was demonstrated that the suppression of MEPs following SAI was positively correlated with the attenuation of the amplitude of the EEG N100 component (Bikmullina et al., 2009). This finding supports the findings of earlier studies that suggested a cortical origin for the suppression of MEPs in SAI paradigm. Furthermore, the attenuation of N100 is an interesting observation by itself as it may be a further support for a link between N100 and activity of $\mathrm{GABA}_{B}$ receptor mediated inhibitory mechanism. In this regard, in a triple pulse study, it was demonstrated that SAI had an inhibitory effect on LICI (Udupa et al., 2009). As previously mentioned, suppression of MEPs in LICI has been associated with the activity of slow acting GABAB receptors. 
Therefore, the attenuation of N100 component following SAI is a further support for the inhibitory effect of SAI on GABAergic mechanism and the plausible involvement of GABA receptors in generation of N100 component.

Moreover, spectral analysis of EEG response following SAI in rat somatosensory cortex has demonstrated that the extent of SAI varies with the cortical state and the frequency of the underlying cortical oscillations. In particular SAI was enhanced following increases in delta oscillations (Funke \& Benali, 2009). The findings of this animal experiment provides further support to reiterate the importance of online EEG recording during TMS-EMG, as some of the inter-subject and inter-laboratory variance that are observed across studies may be explained by the dependence of TMS-EMG measures on cortical states and cortical oscillatory activity immediately before or at the time of TMS stimulation.

\subsection{Repetitive TMS}

A key advantage of concurrent EEG recording is the ability to directly evaluate the effect of rTMS on cortical excitability. As previously mentioned, slow and fast frequency rTMS applied to the motor cortex may have a differential effect on the cortical excitability. As described earlier, the alteration of cortical excitability by rTMS is conventionally probed through evaluating the changes in MEP responses to single pulse of TMS applied to the motor cortex before and after rTMS session. However, brain is a network of interconnected cortical regions and it is unlikely that rTMS effect would remain solely within the stimulated region. In fact, as described above, the TMS induced cortical evoked activity propagates across cortical mantels and may differentially affect the interconnected local and distant brain regions (Ferreri et al., 2010; Voineskos et al., 2010). Furthermore, evaluation of MEP modulations is limited to investigating the cortical activity of the motor cortex while rTMS treatment is often administered in non-motor regions of the cortex such as the DLPFC in the treatment of depression. Finally, the origin of rTMS mediated modulation of MEP remains a matter of debate. Therefore, recording both cortical and peripheral response before, during and after rTMS would enhance our understanding of the effect of rTMS on both motor and non-motor regions of the cortex and would be critical in examining the exact mechanisms through which rTMS exerts its therapeutic effect. In this vein, research examining the cortico-peripheral effect of rTMS is expanding, some of which will be discussed here.

In one of the earlier studies examining the cortical signature of rTMS induced MEP potentiation, the cortical and peripheral response to single pulse TMS were evaluated before and after $5 \mathrm{~Hz}$ rTMS of motor cortex in healthy human subjects (Esser et al., 2006). It was reported that following rTMS, the TMS induced EEG components in response to subthreshold single pulse TMS were selectively potentiated within a time window of 15 to $55 \mathrm{~ms}$. In a similar study, the effect of slow frequency rTMS $(0.6 \mathrm{~Hz})$ was assessed by examining the amplitude of EEG N45 response to subthreshold single pulse TMS before and after 15 minutes of rTMS administration (Van Der Werf \& Paus, 2006). In this study, it was reported that despite the lack of any rTMS induced MEP modulation, the amplitude of N45 response was reduced. This finding may suggest that EEG response is a more sensitive indicator of rTMS effect as compared to MEP. In another study, the effect of $1 \mathrm{~Hz}$ rTMS on cortical oscillations was examined and compared with the suppression of MEPs at the periphery (Brignani et al., 2008). In this study, three 10-min blocks of $1 \mathrm{~Hz}$ rTMS was applied to the left primary motor cortex of healthy subjects. Spectral analysis was performed and the power of alpha and beta band oscillations was obtained during each three rTMS blocks. It was shown that the power of cortical oscillations, more prominently alpha oscillations, increased with increase in TMS 
duration. The increase in power of cortical oscillations was inversely correlated with the suppression of MEP amplitudes at the periphery. It was suggested that the increase of oscillatory activity may be a representation of altered cortical excitability, possibly related to an increase in the activity of inhibitory mechanism (Brignani et al., 2008). Interestingly, the increase in alpha and beta band cortical oscillations was also documented in a recent study that examined cortical response to $20 \mathrm{~Hz}$ rTMS applied to the left primary motor cortex (Veniero et al., 2011). It was demonstrated that synchronization of alpha and beta band cortical oscillations recorded from the $\mathrm{C} 3$ electrode was also positively related to the number of delivered stimuli. Furthermore, there was an inverse correlation between the MEP amplitude and synchronization of beta band oscillations, showing that smaller MEP amplitudes were related to higher power of beta oscillations (Veniero et al., 2011).

Theta burst stimulation is a relatively new rTMS approach that has attracted a lot of interest due to its long lasting effect relative to the short administration period. While 25 minutes of $1 \mathrm{~Hz}$ rTMS may induce changes lasting for about 30 minutes, only 40 seconds of TBS may result in MEP modulation lasting for more than 60 minutes (Huang et al., 2005). Previous TMS-EMG studies had shown that application of cTBS over the motor cortex results in suppression of MEPs at the periphery. Through combination of TMS-EMG with concurrent EEG recording, it has been demonstrated that the cortico-peripheral effect of cTBS involves a reduction in the cortico-muscular coherence within the cortical beta band oscillations measured thorough EEG C3 electrode in the primary motor cortex (Saglam et al., 2008).

The effect of PAS on cortical evoked potentials has recently been investigated to also examine the cortical origin and EEG substrate of PAS induced LTP in the motor cortex of healthy subjects. In an interesting study by Hubert et al it was shown that the EEG changes following PAS, as opposed to MEP changes, could predict the modification of slow wave (0.5-4.7 Hz) activity during sleep (Huber et al., 2008). In this study, PAS was administered to healthy subjects at two ISIs of $10 \mathrm{~ms}$ and $25 \mathrm{~ms}$. Authors then evaluated the cortical evoked potentials in response to single pulse of TMS applied to the motor cortex before and after PAS intervention. Subjects were then allowed to sleep shortly after PAS and their sleep EEG was recorded and analyzed. It was reported that on average PAS at $25 \mathrm{~ms}$ resulted in potentiation of TMS-evoked MEPs and PAS at $10 \mathrm{~ms}$ resulted in suppression of MEP responses, although the reverse was also observed in a few subjects. The study yielded three interesting findings. First, it was shown that there was a weak but significant correlation between the MEP amplitudes measured through EMG and the cortical evoked potentials measured through EEG. Second, interestingly, the location of maximum cortical activation was over the sensorimotor cortex near the site of stimulation where the effect of afferent and cortical stimulation would most likely overlap, thus further supporting the LTP like mechanisms that underlie PAS-induced MEP potentiation. Finally, in subjects that showed a potentiation of cortical evoked potentials in response to PAS intervention (regardless of the ISI or MEP modulation) the power of slow wave activity was increased during sleep that followed the PAS intervention. By contrast, in subjects that cortical evoked potentials were suppressed following PAS intervention (again, regardless of the ISI or MEP modulation), a relative reduction of slow wave activity was observed. The finding that the modulation of cortical evoked potentials was a better predictor of subsequent changes during sleep is a further support for the significance of probing plasticity and modulation of cortical excitability directly from the cortex.

Furthermore, utilizing the cortical evoked potentials to index PAS induced LTP and LTD like plasticity, a recent study has attempted to examine the PAS induced plasticity in both 
the motor cortex and DLPFC of healthy subjects. Application of PAS in the DLPFC was achieved by pairing the afferent peripheral stimulation with single pulse TMS applied to the contralateral DLPFC. The preliminary results of this study, currently presented in an abstract form (Rajji TK et al., 2011), shows that in line with findings of Hubert et al. following PAS intervention in the motor cortex, there was a significant correlation between the cortical evoked potentials recorded at the stimulation site and the MEP response at the periphery. It was noted that while all subjects demonstrated potentiation of cortical evoked potentials, MEPs were not potentiated in a subset of subjects. Finally, it was shown that PAS applied to the DLPFC results in significant potentiation of cortical evoked potentials recorded from the DLPFC which is perhaps, if not the first, one of the first studies directly probing LTP like plasticity in the DLPFC of healthy subjects, a cortical region more closely associated with learning and memory.

Similarly, the effect of rTMS on non-motor regions of the cortex can also be evaluated through TMS-EEG. The observation that LICI modulated cortical oscillations differently in the motor compared to the prefrontal cortex (Farzan et al., 2009) suggests that fast versus slow rTMS and cTBS versus iTBS may have differential modulatory effect on cortical oscillations of motor compared to non-motor regions of the cortex such as DLPFC. In fact, in a recent study published by our group it was demonstrated that one session of $20 \mathrm{~Hz}$ rTMS applied bilaterally to the left and right DLPFC results in increased power of gamma cortical oscillations during cognitive performance in healthy subjects (Barr et al., 2009) and reduced gamma oscillations in patients with schizophrenia (Barr et al. 2011). Future studies should more systematically investigate the effect of rTMS with different stimulation parameters (e.g. frequency) on motor and non-motor regions of the cortex. Such studies would enhance the efficacy of rTMS therapy.

\section{Reproducibility and reliability of TMS-EEG-EMG measures}

Research experiments systematically examining the test-retest reliability of TMS induced MEPs are relatively scarce. In this regard, one study has examined the reliability of TMS in identifying the APB muscle motor map between two separate sessions (Corneal et al., 2005). It was also shown that increasing the number of delivered stimuli in TMS paradigms would reduce the intersession variability (Boroojerdi et al., 2000). There are also a few studies that have demonstrated the reliability of TMS induced MEP modulation in the paired pulse paradigms of SICI and ICF (Boroojerdi et al., 2000; Maeda et al., 2002). For example, Maeda et al. reported that SICI was reproducible and correlated between two sessions separated by two weeks (Maeda et al., 2002).

Given the increasing popularity of TMS-EEG-EMG approach as both a therapeutic and diagnostic tool, the number of research experiments examining the reliability and validity of cortical and peripheral indices of TMS is on the rise. The reproducibility of TMS induced cortical responses to single pulse TMS were recently demonstrated in the motor cortex and DLPFC of healthy subjects (Lioumis et al., 2009). Lioumis et al. reported generation of six EEG peaks in the ipsilateral (at latencies 13, 32, 54, 66, 111, 172 ms post TMS) and contralateral $(12,31,50,73,111,176$ ms post TMS) motor cortex following the application of suprathreshold single pulse to the left motor cortex, and six peaks were defined in the ipsilateral DLPFC (first one contaminated by noise, 25, 49, 64, 113, $170 \mathrm{~ms}$ post TMS) and contralateral DLPFC $(21,32,48,63,113$, and $174 \mathrm{~ms}$ post TMS) following suprathreshold TMS to the left DLPFC. It was reported that the amplitudes of TMS evoked cortical 
potentials at various stimulation intensities were highly reliable in both the ipsilateral DLPFC and motor cortex between sessions separated by a one-week interval (test-retest correlation >0.83), while considering the contralateral response, contralateral DLPFC response was more reliable than motor cortex perhaps reflecting the stronger interhemispheric connectivity of the prefrontal cortices (Lioumis et al., 2009).

In a study conducted by our group, we extended the results of previous reports by demonstrating the reliability of cortical and peripheral indices of LICI, as well as the reliability of LICI mediated modulation of each cortical oscillation in both the left motor cortex and left DLPFC (Farzan et al., 2010b). In the motor cortex, we showed that all EEG and EMG measures of LICI were highly reliable, and in the DLPFC all EEG indices of LICI were highly reliable between sessions separated by one-week (Cronbach's alpha ranging from 0.7 to 0.9 ). Furthermore, we replicated the correlation between EEG measures of LICI and EMG measures of LICI in an extended sample size of 36 subjects. Finally, for the first time we demonstrated a positive correlation between the EEG measures of LICI and the duration of cortical silent period, as indexed through the CSP paradigm. Previous pharmacological studies had linked the cortical silent period and MEP suppression by LICI to the activity of $\mathrm{GABA}_{\mathrm{B}}$ receptor mediated inhibitory neurotransmission. Collectively, these findings further confirm the validity and reliability of EEG measures of LICI as indices of GABAergic inhibitory mechanism, and also provide evidence for the cortical origin of MEP modulation and the silent period in the LICI and CSP paradigms, respectively. In the next section, we will discuss how some of these measures can be used to enhance the diagnostic and therapeutic efficacy of TMS.

\section{TMS-EEG-EMG in diagnosis and treatment of neuropsychiatric disorders}

Since the introduction of TMS in 1985, deficits of TMS induced MEPs have been reported in a number of neurological and psychiatric conditions. Among movement disorders, reduction of SICI (Hanajima et al., 1996; Pierantozzi et al., 2001; Ridding et al., 1995; Strafella et al., 2000), prolongation of silent period, and increases in LICI has been reported in the motor cortex of patients with Parkinson's disease (Berardelli et al., 2008; Berardelli et al., 1996). In another study, impairment of both SICI and ICF was observed in early Parkinson's disease patients not treated with dopamine agonists (Bares et al., 2003). Among neuropsychiatric disorders, reduction of CSP, IHI and SICI was reported in unmedicated patients with schizophrenia, while these measures of cortical inhibition were not significantly impaired in medicated patients with schizophrenia (Daskalakis et al., 2002a). Similarly, the reduction of SICI, IHI and CSP was also demonstrated in the motor cortex of patients with bipolar disorder (Levinson et al., 2007). Application of TMS-EMG in cognitive disorders has shown that patients with Alzheimer's disease have reduced SAI measures (Di Lazzaro et al., 2002b), and it was reported that an oral dose of rivastigmine enhanced SAI in a subgroup of patients (Di Lazzaro et al., 2002b). Reduction of SAI has also been reported in early Alzheimer's disease (Nardone et al., 2008). While these TMS-EMG indices have moved us a step closer to the underlying source of some of these disorders, the exact mechanisms underlying the generation of MEPs and thus the sources of such impairments have remained a matter of debate. Furthermore, the pathophysiology of neuropsychiatric illnesses such as schizophrenia, bipolar disorder and Alzheimer's disease is more closely associated with impairment of non-motor regions of the cortex which are not easily accessible through the conventional TMS-EMG indices of cortical processes. 
The combination of TMS-EMG with concurrent EEG recordings permits the assessment of cortical origin of MEP impairments. For example, the cortico-muscular coherence and EEG spectral analysis, discussed in previous sections, can be used to understand the origin of MEP impairments in movement disorders and neurological diseases that have abnormalities of the corticospinal pathway and the cortical oscillatory activity of the motor cortex such as in Parkinson's disease and dystonia (Di Lazzaro et al., 2009; Levy et al.). Furthermore, the combination of TMS with EEG allows for examining cortical processes in neuropsychiatric disorders which are closely associated with impairments of cortical oscillatory activity in the non-motor regions of the cortex. For example, impairments in gamma oscillations have been reported during cognitive performance in the prefrontal cortex in patients with schizophrenia (Basar-Eroglu et al., 2007; Cho et al., 2006). In this regard, we have recently replicated previous studies, such as those by Basar-Eroglu et al. by demonstrating that patients with schizophrenia exhibit excessive power of gamma oscillations during working memory performance (Barr et al., 2010). Given these frontal gamma deficits, we conducted a TMS-EEG-EMG experiment to examine the integrity of LICI induced modulation of gamma oscillations in the DLPFC of patients with schizophrenia compared to healthy subject in whom, as described previously, LICI resulted in a significant inhibition of gamma oscillations following paired pulse stimulation of DLPFC(Farzan et al., 2009). Utilizing these EEG measures of LICI, which were shown to have high test-retest reliability (Farzan et al., 2010b), we have in fact demonstrated that inhibition of gamma oscillations was selectively impaired in the DLPFC of patients with schizophrenia compared to both healthy subjects and patients with bipolar disorder (Farzan et al., 2010a). Interestingly, we observed no deficits in the EEG or EMG measures of LICI in the motor cortex or in modulation of any other frequency bands in the DLPFC. Patients with bipolar disorder were similar to patients with schizophrenia in relation to severity of symptoms, illness duration, and history of psychosis, and about half of them were on antipsychotic medications. In addition, the extent of gamma inhibition did not correlate with the medication dosage, suggesting that the specificity of gamma inhibition deficits to schizophrenia and the DLPFC is less likely to be part of a generalized deficit that is simply related to psychotropic medications and it may represent a candidate endophenotype for schizophrenia. Combining these finding with evidence of impaired gamma modulation during cognitive performance in patients with schizophrenia, it may be hypothesized that the impairments of LICI induced modulation of gamma oscillations in the DLPFC may, at least partly, underlie the excessive power of gamma oscillations that has been reported during working memory performance and perhaps the frontal cognitive deficits in this illness.

In line with these findings, cognitive impairments and the deficits in learning and memory, a process thought to be related to cortical plasticity, constitute one of the core deficits of schizophrenia (Gold \& Harvey, 1993). Indeed, in a previous TMS-EMG study, TMS paradigm PAS was used to investigate the integrity of LTP-like plasticity in the motor cortex of patients with schizophrenia (Frantseva et al., 2008). In this study, the effect of PAS in patients with schizophrenia and healthy subjects was evaluated through evaluating the TMS induced MEP response to single pulse of TMS after PAS, and also through examining the effect of PAS on learning a subsequent motor task (rotary pursuit motor task). It was demonstrated that not only patients with schizophrenia had significantly less MEP facilitation in response to PAS, but there was also a correlation between the PAS induced potentiation of MEPs and performance on the motor learning task (Frantseva et al., 2008). That is, patients with schizophrenia who had less MEP potentiation following PAS compared to healthy subjects, also performed poorly on the subsequent motor task 
compared to healthy subjects. Consistent with these finding, both medicated and unmedicated patients with schizophrenia demonstrated a reduced plastic response to 15 min of $1 \mathrm{~Hz}$ rTMS applied to the motor cortex compared to healthy subjects (Fitzgerald et al., 2004). It was also shown that the duration of CSP at baseline correlated positively with the rTMS response. That is, patients who had less plastic response to rTMS also had a shorter CSP duration at baseline. The results of these studies would be significantly complemented by examining LTP like plasticity in the DLPFC of patients with schizophrenia through PAS or rTMS combined with EEG. In fact, such experiments are currently underway. Preliminary results of a recent experiment has so far shown that following application of PAS in the DLPFC, patients with schizophrenia demonstrate a deficit in potentiation of cortical evoked potentials recorded from the DLPFC compared to the healthy subjects (Rajji TK et al., 2011).

Furthermore, similar to PAS and LICI, EEG measures of SAI can be used to assess the cortical origin and the cortical oscillatory activity underlying the SAI deficits in Alzheimer's disease. The combination of TMS-EMG with EEG would further permit assessing the effect of afferent sensory stimuli on the prefrontal cortex, which is perhaps more closely associated with the impairment of cognitive disorders. Similar to PAS, this can be conducted through combination of peripheral nerve and single pulse TMS applied to the DLPFC, as opposed to the motor cortex, and examining the amplitude and frequency components of the cortical evoked activity in the DLPFC as well as the spread of the signal to the interconnected cortical regions.

Finally, the neurophysiological measures obtained through TMS-EEG-EMG studies would significantly enhance our understanding of the mechanism through which repetitive TMS exerts its effect, and would further enable us to optimally select stimulation parameters that would benefit individual patients based on their underlying impairment. To this day, the outcomes of repetitive TMS therapy have been inconsistent across studies. There are several reasons for this variability. First, certain stimulation patterns such as intensity, frequency, and number of stimuli delivered can significantly influence the outcome measures. Second, emerging lines of evidence suggest that rTMS effects are highly state dependent (Silvanto et al., 2008a; Silvanto et al., 2008b). That is the level of underlying cortical activity prior to rTMS treatment may influence the rTMS effect and could even be used to predict whether rTMS would suppress or facilitate cortical excitability. However, the classical rTMS treatments, not equipped with any concurrent EEG recordings, have not evaluated or controlled such baseline activities, which explains some of the variability observed in the rTMS studies.

Therefore, online EEG recording during rTMS therapy can improve the efficacy of TMS therapy in several ways. First, proof of concept studies similar to some of the rTMS-EEG experiments described before (Barr et al., 2010; Esser et al., 2006; Saglam et al., 2008; Van Der Werf et al., 2006) should be expanded to more systematically examine the effect of various stimulation parameters (e.g. intensity and frequency) on the cortical evoked potentials that seem to be a better predictor of subtle changes that take place in the cortex, as compared to the conventional MEP responses (Farzan et al., 2010b; Ferreri et al., 2010; Huber et al., 2008; Van Der Werf \& Paus, 2006). Second, the results of these studies can then be utilized to custom design rTMS treatment sessions though carefully selecting parameters that would induce a specific response in the cortex (e.g. increased or reduced cortical excitability) similar to the tACS frequency adjustments in a recent study by Schutter and Hortensius (Schutter \& Hortensius, 2011). Finally, a major advancement in EEG and TMS technology would hopefully give rise to the design of reliable EEG triggered TMS machines in which continuous online monitoring of underlying cortical potential would trigger the rTMS 
stimulation whenever the cortical activity reaches a state which would be more prone to be affected by the therapeutic efficacy of a specific rTMS therapy (e.g. low vs. fast frequency) (Rotenberg, 2009). Finally, in an even more advanced design, an EEG-triggered TMS system would be capable of updating the rTMS parameters (e.g. changing the rTMS frequency) during the treatment and based on the online analysis of changes in the cortical activity. Such advances in cortical stimulation technology could revolutionize the TMS field and would be a major step toward making the cortical stimulation a standard treatment approach for several neuropsychiatric disorders.

\section{Conclusion}

Advances in cortical stimulation and cortical recording techniques over the past few decades have allowed researchers to more systematically and non-invasively investigate the neurophysiological processes from the intact cortex of awake humans. Among such advancements, the concurrent TMS and EMG recordings has been instrumental in identifying and probing cortical processes that underlie generation and modulation of motor evoked potentials. The ability to evaluate cortical processes such as inhibition, excitation and plasticity in healthy subjects has further led to the discovery of pathophysiological processes in various neuropsychiatric disorders. In recent years, the introduction of TMS compatible EEG systems has brought about a significant advancement to the TMS field, enhancing both the diagnostic and therapeutic aspects of TMS. This is achieved through the superior temporal, spatial and spectral precision of TMS-EEG combination in identifying subtle changes of cortical activity before, during and post TMS delivery in both motor and non-motor regions of the cortex. In addition, the integration of EEG data with EMG recordings has provided further confirmation for the cortical origin of the classical TMS-EMG paradigms and has provided new information about the cortical oscillatory activity that underlies the generation and modulation of the peripheral effects. Although today there are only a few number of studies that have systematically investigated the reliability of EEG measures of TMS, the results are promising and suggest that TMS does have a potential for reliably identifying biological markers for several neuropsychiatric disorders. In regards to the future prospect of TMS-EEG-EMG research, it is expected that the integration of online EEG recording during rTMS therapy and advancements of EEG triggered cortical stimulation technology would significantly enhance the therapeutic outcomes of TMS and increase the certainty with which its therapeutic efficacy is predicated for each clinical condition. Such advancement would ultimately assist physicians in selecting patients who would benefit from rTMS therapy.

\section{References}

Adrian ED, Moruzzi G. (1939). Impulses in the pyramidal tract. J Physiol, 97, pp 153-199

Anand S, Hotson J. (2002). Transcranial magnetic stimulation: neurophysiological applications and safety. Brain Cogn, 50, pp 366-386

Bares M, Kanovsky P, Klajblova H, Rektor I. (2003). Intracortical inhibition and facilitation are impaired in patients with early Parkinson's disease: a paired TMS study. Eur J Neurol, 10, pp 385-389

Barker AT, Jalinous R, Freeston IL. (1985). Non-invasive magnetic stimulation of human motor cortex. Lancet, 1, pp 1106-1107 
Barr MS, Farzan F, Arenovich T, Chen R, Fitzgerald PB, Daskalakis ZJ. (2011). The effect of repetitive transcranial magnetic stimulation on gamma oscillatory activity in schizophrenia. PLoS One, 6, p e22627

Barr MS, Farzan F, Rusjan PM, Chen R, Fitzgerald PB, Daskalakis ZJ. (2009). Potentiation of gamma oscillatory activity through repetitive transcranial magnetic stimulation of the dorsolateral prefrontal cortex. Neuropsychopharmacology, 34, pp 2359-2367

Barr MS, Farzan F, Tran LC, Chen R, Fitzgerald PB, Daskalakis ZJ. (2010). Evidence for excessive frontal evoked gamma oscillatory activity in schizophrenia during working memory. Schizophr Res, 121, pp 146-152

Basar-Eroglu C, Brand A, Hildebrandt H, Karolina Kedzior K, Mathes B, Schmiedt C. (2007). Working memory related gamma oscillations in schizophrenia patients. Int J Psychophysiol, 64, pp 39-45

Bender S, Basseler K, Sebastian I, Resch F, Kammer T, Oelkers-Ax R, et al. (2005). Electroencephalographic response to transcranial magnetic stimulation in children: Evidence for giant inhibitory potentials. Ann Neurol, 58, pp 58-67

Berardelli A, Abbruzzese G, Chen R, Orth M, Ridding MC, Stinear C, et al. (2008). Consensus paper on short-interval intracortical inhibition and other transcranial magnetic stimulation intracortical paradigms in movement disorders. Brain Stimul, 1, pp 183-191

Berardelli A, Rona S, Inghilleri M, Manfredi M. (1996). Cortical inhibition in Parkinson's disease. A study with paired magnetic stimulation. Brain, 119 ( $\mathrm{Pt} 1)$, pp 71-77

Bikmullina R, Kicic D, Carlson S, Nikulin VV. (2009). Electrophysiological correlates of short-latency afferent inhibition: a combined EEG and TMS study. Exp Brain Res, 194, pp 517-526

Boroojerdi B, Kopylev L, Battaglia F, Facchini S, Ziemann U, Muellbacher W, et al. (2000). Reproducibility of intracortical inhibition and facilitation using the paired-pulse paradigm. Muscle Nerve, 23, pp 1594-1597

Bragin A, Jando G, Nadasdy Z, Hetke J, Wise K, Buzsaki G. (1995). Gamma (40-100 Hz) oscillation in the hippocampus of the behaving rat. J Neurosci, 15, pp 47-60

Brignani D, Manganotti P, Rossini PM, Miniussi C. (2008). Modulation of cortical oscillatory activity during transcranial magnetic stimulation. Hum Brain Mapp, 29, pp 603-612

Brown P, Salenius S, Rothwell JC, Hari R. (1998). Cortical correlate of the Piper rhythm in humans. J Neurophysiol, 80, pp 2911-2917

Buonomano DV, Merzenich MM. (1998). Cortical plasticity: from synapses to maps. Annu Rev Neurosci, 21, pp 149-186

Buzsáki G (2006). Rhythms of the Brain, Oxford University Press, 13 978-0-19-530106-9, New York

Chen R. (2004). Interactions between inhibitory and excitatory circuits in the human motor cortex. Exp Brain Res, 154, pp 1-10

Chen R, Classen J, Gerloff C, Celnik P, Wassermann EM, Hallett M, et al. (1997). Depression of motor cortex excitability by low-frequency transcranial magnetic stimulation. Neurology, 48, pp 1398-1403

Chen R, Lozano AM, Ashby P. (1999). Mechanism of the silent period following transcranial magnetic stimulation. Evidence from epidural recordings. Exp Brain Res, 128, pp 539-542

Chen R, Yung D, Li JY. (2003). Organization of ipsilateral excitatory and inhibitory pathways in the human motor cortex. J Neurophysiol, 89, pp 1256-1264

Cho RY, Konecky RO, Carter CS. (2006). Impairments in frontal cortical gamma synchrony and cognitive control in schizophrenia. Proc Natl Acad Sci U S A, 103, pp 19878-19883 
Classen J, Wolters A, Stefan K, Wycislo M, Sandbrink F, Schmidt A, et al. (2004). Paired associative stimulation. Suppl Clin Neurophysiol, 57, pp 563-569

Corneal SF, Butler AJ, Wolf SL. (2005). Intra- and intersubject reliability of abductor pollicis brevis muscle motor map characteristics with transcranial magnetic stimulation. Arch Phys Med Rehabil, 86, pp 1670-1675

Daskalakis ZJ, Christensen BK, Chen R, Fitzgerald PB, Zipursky RB, Kapur S. (2002a). Evidence for impaired cortical inhibition in schizophrenia using transcranial magnetic stimulation. Arch Gen Psychiatry, 59, pp 347-354

Daskalakis ZJ, Christensen BK, Fitzgerald PB, Roshan L, Chen R. (2002b). The mechanisms of interhemispheric inhibition in the human motor cortex. J Physiol, 543, pp 317-326

Daskalakis ZJ, Farzan F, Barr MS, Maller JJ, Chen R, Fitzgerald PB. (2008). Long-interval cortical inhibition from the dorsolateral prefrontal cortex: a TMS-EEG study. Neuropsychopharmacology, 33, pp 2860-2869

Daskalakis ZJ, Moller B, Christensen BK, Fitzgerald PB, Gunraj C, Chen R. (2006). The effects of repetitive transcranial magnetic stimulation on cortical inhibition in healthy human subjects. Exp Brain Res, 174, pp 403-412

Daskalakis ZJ, Paradiso GO, Christensen BK, Fitzgerald PB, Gunraj C, Chen R. (2004). Exploring the connectivity between the cerebellum and motor cortex in humans. $J$ Physiol, 557, pp 689-700

Day BL, Dressler D, Maertens de Noordhout A, Marsden CD, Nakashima K, Rothwell JC, et al. (1989). Electric and magnetic stimulation of human motor cortex: surface EMG and single motor unit responses. J Physiol, 412, pp 449-473

Di Lazzaro V, Dileone M, Pilato F, Capone F, Musumeci G, Ranieri F, et al. (2011). Modulation of motor cortex neuronal networks by rTMS: comparison of local and remote effects of six different protocols of stimulation. J Neurophysiol, 105, pp 21502156

Di Lazzaro V, Oliviero A, Mazzone P, Insola A, Pilato F, Saturno E, et al. (2001). Comparison of descending volleys evoked by monophasic and biphasic magnetic stimulation of the motor cortex in conscious humans. Exp Brain Res, 141, pp 121-127

Di Lazzaro V, Oliviero A, Mazzone P, Pilato F, Saturno E, Insola A, et al. (2002a). Direct demonstration of long latency cortico-cortical inhibition in normal subjects and in a patient with vascular parkinsonism. Clin Neurophysiol, 113, pp 1673-1679

Di Lazzaro V, Oliviero A, Meglio M, Cioni B, Tamburrini G, Tonali P, et al. (2000). Direct demonstration of the effect of lorazepam on the excitability of the human motor cortex. Clin Neurophysiol, 111, pp 794-799

Di Lazzaro V, Oliviero A, Pilato F, Saturno E, Dileone M, Mazzone P, et al. (2004). The physiological basis of transcranial motor cortex stimulation in conscious humans. Clin Neurophysiol, 115, pp 255-266

Di Lazzaro V, Oliviero A, Profice P, Dileone M, Pilato F, Insola A, et al. (2009). Reduced cerebral cortex inhibition in dystonia: direct evidence in humans. Clin Neurophysiol, 120, pp 834-839

Di Lazzaro V, Oliviero A, Profice P, Insola A, Mazzone P, Tonali P, et al. (1999a). Direct demonstration of interhemispheric inhibition of the human motor cortex produced by transcranial magnetic stimulation. Exp Brain Res, 124, pp 520-524

Di Lazzaro V, Oliviero A, Profice P, Insola A, Mazzone P, Tonali P, et al. (1999b). Direct recordings of descending volleys after transcranial magnetic and electric motor cortex stimulation in conscious humans. Electroencephalogr Clin Neurophysiol Suppl, 51 , pp 120-126 
Di Lazzaro V, Oliviero A, Saturno E, Dileone M, Pilato F, Nardone R, et al. (2005). Effects of lorazepam on short latency afferent inhibition and short latency intracortical inhibition in humans. J Physiol, 564, pp 661-668

Di Lazzaro V, Oliviero A, Tonali PA, Marra C, Daniele A, Profice P, et al. (2002b). Noninvasive in vivo assessment of cholinergic cortical circuits in AD using transcranial magnetic stimulation. Neurology, 59, pp 392-397

Di Lazzaro V, Pilato F, Dileone M, Profice P, Ranieri F, Ricci V, et al. (2007). Segregating two inhibitory circuits in human motor cortex at the level of GABAA receptor subtypes: a TMS study. Clin Neurophysiol, 118, pp 2207-2214

Di Lazzaro V, Restuccia D, Oliviero A, Profice P, Ferrara L, Insola A, et al. (1998). Magnetic transcranial stimulation at intensities below active motor threshold activates intracortical inhibitory circuits. Exp Brain Res, 119, pp 265-268

Di Lazzaro V, Rothwell JC, Oliviero A, Profice P, Insola A, Mazzone P, et al. (1999c). Intracortical origin of the short latency facilitation produced by pairs of threshold magnetic stimuli applied to human motor cortex. Exp Brain Res, 129, pp 494-499

Engel AK, Fries P. (2010). Beta-band oscillations--signalling the status quo? Curr Opin Neurobiol, 20, pp 156-165

Engel AK, Singer W. (2001). Temporal binding and the neural correlates of sensory awareness. Trends Cogn Sci, 5, pp 16-25

Esser SK, Huber R, Massimini M, Peterson MJ, Ferrarelli F, Tononi G. (2006). A direct demonstration of cortical LTP in humans: A combined TMS/EEG study. Brain Res Bull, 69, pp 86-94

Farzan F, Barr MS, Levinson AJ, Chen R, Wong W, Fitzgerald PB, et al. (2010a). Evidence for gamma inhibition deficits in the dorsolateral prefrontal cortex of patients with schizophrenia. Brain, 133, pp 1505-1514

Farzan F, Barr MS, Levinson AJ, Chen R, Wong W, Fitzgerald PB, et al. (2010b). Reliability of Long Interval Cortical Inhibition in Healthy Human Subjects: A TMS-EEG Study. J Neurophysiol,

Farzan F, Barr MS, Wong W, Chen R, Fitzgerald PB, Daskalakis ZJ. (2009). Suppression of gamma-oscillations in the dorsolateral prefrontal cortex following long interval cortical inhibition: a TMS-EEG study. Neuropsychopharmacology, 34, pp 1543-1551

Ferbert A, Priori A, Rothwell JC, Day BL, Colebatch JG, Marsden CD. (1992). Interhemispheric inhibition of the human motor cortex. J Physiol, 453, pp 525-546

Ferreri F, Pasqualetti P, Maatta S, Ponzo D, Ferrarelli F, Tononi G, et al. (2010). Human brain connectivity during single and paired pulse transcranial magnetic stimulation. Neuroimage, 54, pp 90-102

Fitzgerald PB, Brown TL, Marston NA, Oxley T, De Castella A, Daskalakis ZJ, et al. (2004). Reduced plastic brain responses in schizophrenia: a transcranial magnetic stimulation study. Schizophr Res, 71, pp 17-26

Fitzgerald PB, Fountain S, Daskalakis ZJ. (2006). A comprehensive review of the effects of rTMS on motor cortical excitability and inhibition. Clin Neurophysiol, 117, pp 25842596

Frantseva MV, Fitzgerald PB, Chen R, Moller B, Daigle M, Daskalakis ZJ. (2008). Evidence for impaired long-term potentiation in schizophrenia and its relationship to motor skill learning. Cereb Cortex, 18, pp 990-996

Fries P, Nikolic D, Singer W. (2007). The gamma cycle. Trends Neurosci, 30, pp 309-316

Funke K, Benali A. (2009). Short-latency afferent inhibition varies with cortical state in rat somatosensory cortex. Neuroreport, 20, pp 1313-1318 
Gerloff C, Cohen LG, Floeter MK, Chen R, Corwell B, Hallett M. (1998). Inhibitory influence of the ipsilateral motor cortex on responses to stimulation of the human cortex and pyramidal tract. J Physiol, 510 ( Pt 1), pp 249-259

Gold JM, Harvey PD. (1993). Cognitive deficits in schizophrenia. Psychiatr Clin North Am, 16, pp 295-312

Hallett M. (2000). Transcranial magnetic stimulation and the human brain. Nature, 406, pp $147-150$

Hanajima R, Ugawa Y, Terao Y, Ogata K, Kanazawa I. (1996). Ipsilateral cortico-cortical inhibition of the motor cortex in various neurological disorders. J Neurol Sci, 140, pp 109-116

Howard MW, Rizzuto DS, Caplan JB, Madsen JR, Lisman J, Aschenbrenner-Scheibe R, et al. (2003). Gamma oscillations correlate with working memory load in humans. Cereb Cortex, 13, pp 1369-1374

Huang YZ, Edwards MJ, Rounis E, Bhatia KP, Rothwell JC. (2005). Theta burst stimulation of the human motor cortex. Neuron, 45, pp 201-206

Huber R, Maatta S, Esser SK, Sarasso S, Ferrarelli F, Watson A, et al. (2008). Measures of cortical plasticity after transcranial paired associative stimulation predict changes in electroencephalogram slow-wave activity during subsequent sleep. J Neurosci, 28, pp 7911-7918

Hutcheon B, Yarom Y. (2000). Resonance, oscillation and the intrinsic frequency preferences of neurons. Trends Neurosci, 23, pp 216-222

Ilmoniemi RJ, Kicic D. (2009). Methodology for combined TMS and EEG. Brain Topogr, 22, pp 233-248

Ilmoniemi RJ, Virtanen J, Ruohonen J, Karhu J, Aronen HJ, Naatanen R, et al. (1997). Neuronal responses to magnetic stimulation reveal cortical reactivity and connectivity. Neuroreport, 8, pp 3537-3540

Inghilleri M, Berardelli A, Cruccu G, Manfredi M. (1993). Silent period evoked by transcranial stimulation of the human cortex and cervicomedullary junction. $J$ Physiol, 466, pp 521-534.

Kahkonen S, Kesaniemi M, Nikouline VV, Karhu J, Ollikainen M, Holi M, et al. (2001). Ethanol modulates cortical activity: direct evidence with combined TMS and EEG. Neuroimage, 14, pp 322-328

Kahkonen S, Wilenius J. (2007). Effects of alcohol on TMS-evoked N100 responses. J Neurosci Methods, 166, pp 104-108

Kahkonen S, Wilenius J, Nikulin VV, Ollikainen M, Ilmoniemi RJ. (2003). Alcohol reduces prefrontal cortical excitability in humans: a combined TMS and EEG study. Neuropsychopharmacology, 28, pp 747-754

Kanai R, Chaieb L, Antal A, Walsh V, Paulus W. (2008). Frequency-dependent electrical stimulation of the visual cortex. Curr Biol, 18, pp 1839-1843

Kapogiannis D, Wassermann EM. (2008). Transcranial magnetic stimulation in Clinical Pharmacology. Cent Nerv Syst Agents Med Chem, 8, pp 234-240

Kimiskidis VK, Papagiannopoulos S, Kazis DA, Vasiliadis G, Oikonomidi A, Sotirakoglou $\mathrm{K}$, et al. (2008). Silent period (SP) to transcranial magnetic stimulation: the EEG substrate. Brain Stimulation 1. Abstracts from the 3rd international conference on transcranial magnetic simulation and direct current stimulation, pp 315-316

Knyazev GG. (2007). Motivation, emotion, and their inhibitory control mirrored in brain oscillations. Neurosci Biobehav Rev, 31, pp 377-395 
Komssi S, Aronen HJ, Huttunen J, Kesaniemi M, Soinne L, Nikouline VV, et al. (2002). Ipsiand contralateral EEG reactions to transcranial magnetic stimulation. Clin Neurophysiol, 113, pp 175-184

Komssi S, Kahkonen S. (2006). The novelty value of the combined use of electroencephalography and transcranial magnetic stimulation for neuroscience research. Brain Res Brain Res Rev, 52, pp 183-192

Krnjevic K, Randic M, Straughan DW. (1964). CORTICAL INHIBITION. Nature, 201, pp 1294-1296

Kujirai T, Caramia MD, Rothwell JC, Day BL, Thompson PD, Ferbert A, et al. (1993). Corticocortical inhibition in human motor cortex. J Physiol, 471, pp 501-519

Levinson AJ, Young LT, Fitzgerald PB, Daskalakis ZJ. (2007). Cortical inhibitory dysfunction in bipolar disorder: a study using transcranial magnetic stimulation. J Clin Psychopharmacol, 27, pp 493-497

Levy R, Lozano AM, Lang AE, Dostrovsky JO. (2010). Event-related desynchronization of motor cortical oscillations in patients with multiple system atrophy. Exp Brain Res, 206, pp 1-13

Linkenkaer-Hansen K, Smit DJ, Barkil A, van Beijsterveldt TE, Brussaard AB, Boomsma DI, et al. (2007). Genetic contributions to long-range temporal correlations in ongoing oscillations. J Neurosci, 27, pp 13882-13889

Lioumis P, Kicic D, Savolainen P, Makela JP, Kahkonen S. (2009). Reproducibility of TMSEvoked EEG responses. Hum Brain Mapp, 30, pp 1387-1396

Maeda F, Gangitano M, Thall M, Pascual-Leone A. (2002). Inter- and intra-individual variability of paired-pulse curves with transcranial magnetic stimulation (TMS). Clin Neurophysiol, 113, pp 376-382

Maeda F, Keenan JP, Tormos JM, Topka H, Pascual-Leone A. (2000). Interindividual variability of the modulatory effects of repetitive transcranial magnetic stimulation on cortical excitability. Exp Brain Res, 133, pp 425-430

Maki H, Ilmoniemi RJ. (2010a). EEG oscillations and magnetically evoked motor potentials reflect motor system excitability in overlapping neuronal populations. Clin Neurophysiol, 121, pp 492-501

Maki H, Ilmoniemi RJ. (2010b). The relationship between peripheral and early cortical activation induced by transcranial magnetic stimulation. Neurosci Lett,

McCormick DA. (1989). GABA as an inhibitory neurotransmitter in human cerebral cortex. J Neurophysiol, 62, pp 1018-1027

McDonnell MN, Orekhov Y, Ziemann U. (2006). The role of GABA(B) receptors in intracortical inhibition in the human motor cortex. Exp Brain Res, 173, pp 86-93

Merton PA, Morton HB. (1980). Stimulation of the cerebral cortex in the intact human subject. Nature, 285, p 227

Mima T, Hallett M. (1999). Electroencephalographic analysis of cortico-muscular coherence: reference effect, volume conduction and generator mechanism. Clin Neurophysiol, 110, pp 1892-1899

Nakamura H, Kitagawa H, Kawaguchi Y, Tsuji H. (1997). Intracortical facilitation and inhibition after transcranial magnetic stimulation in conscious humans. J Physiol, 498 ( Pt 3), pp 817-823

Nardone R, Bergmann J, Kronbichler M, Kunz A, Klein S, Caleri F, et al. (2008). Abnormal short latency afferent inhibition in early Alzheimer's disease: a transcranial magnetic demonstration. J Neural Transm, 115, pp 1557-1562 
Neuper C, Pfurtscheller G. (2001). Evidence for distinct beta resonance frequencies in human EEG related to specific sensorimotor cortical areas. Clin Neurophysiol, 112, pp 20842097

Ni Z, Gunraj C, Nelson AJ, Yeh IJ, Castillo G, Hoque T, et al. (2009). Two phases of interhemispheric inhibition between motor related cortical areas and the primary motor cortex in human. Cereb Cortex, 19, pp 1654-1665

Nikulin VV, Kicic D, Kahkonen S, Ilmoniemi RJ. (2003). Modulation of electroencephalographic responses to transcranial magnetic stimulation: evidence for changes in cortical excitability related to movement. Eur J Neurosci, 18, pp 12061212

Palva S, Palva JM. (2007). New vistas for alpha-frequency band oscillations. Trends Neurosci, 30, pp 150-158

Pascual-Leone A, Valls-Sole J, Wassermann EM, Hallett M. (1994). Responses to rapid-rate transcranial magnetic stimulation of the human motor cortex. Brain, 117 ( $\mathrm{Pt} 4$ ), pp 847-858

Pascual-Leone A, Walsh V, Rothwell J. (2000). Transcranial magnetic stimulation in cognitive neuroscience--virtual lesion, chronometry, and functional connectivity. Curr Opin Neurobiol, 10, pp 232-237

Paus T, Sipila PK, Strafella AP. (2001). Synchronization of neuronal activity in the human primary motor cortex by transcranial magnetic stimulation: an EEG study. $J$ Neurophysiol, 86, pp 1983-1990

Pell GS, Roth Y, Zangen A. (2010). Modulation of cortical excitability induced by repetitive transcranial magnetic stimulation: influence of timing and geometrical parameters and underlying mechanisms. Prog Neurobiol, 93, pp 59-98

Pfurtscheller G, Neuper C, Pichler-Zalaudek K, Edlinger G, Lopes da Silva FH. (2000). Do brain oscillations of different frequencies indicate interaction between cortical areas in humans? Neurosci Lett, 286, pp 66-68

Pierantozzi M, Marciani MG, Palmieri MG, Brusa L, Galati S, Caramia MD, et al. (2004). Effect of Vigabatrin on motor responses to transcranial magnetic stimulation: an effective tool to investigate in vivo GABAergic cortical inhibition in humans. Brain Res, 1028, pp 1-8

Pierantozzi M, Palmieri MG, Marciani MG, Bernardi G, Giacomini P, Stanzione P. (2001). Effect of apomorphine on cortical inhibition in Parkinson's disease patients: a transcranial magnetic stimulation study. Exp Brain Res, 141, pp 52-62

Pike FG, Goddard RS, Suckling JM, Ganter P, Kasthuri N, Paulsen O. (2000). Distinct frequency preferences of different types of rat hippocampal neurones in response to oscillatory input currents. J Physiol, $529 \mathrm{Pt} 1$, pp 205-213

Pineda JA. (2005). The functional significance of $\mathrm{mu}$ rhythms: translating "seeing" and "hearing" into "doing". Brain Res Brain Res Rev, 50, pp 57-68

Rajji TK, Sun Y, Farzan F, D'Souza R, Wass C, Mulsant BH, et al. (2011). Assessing plasticity in the dorsolateral prefrontal cortex in patients with schizophrenia. Conference Abstract. The International Congress on Schizophrenia Research, April 2011.

Ridding MC, Rothwell JC. (2007). Is there a future for therapeutic use of transcranial magnetic stimulation? Nat Rev Neurosci, 8, pp 559-567

Ridding MC, Sheean G, Rothwell JC, Inzelberg R, Kujirai T. (1995). Changes in the balance between motor cortical excitation and inhibition in focal, task specific dystonia. $J$ Neurol Neurosurg Psychiatry, 59, pp 493-498

Roopun AK, Kramer MA, Carracedo LM, Kaiser M, Davies CH, Traub RD, et al. (2008). Temporal Interactions between Cortical Rhythms. Front Neurosci, 2, pp 145-154 
Rosanova M, Casali A, Bellina V, Resta F, Mariotti M, Massimini M. (2009). Natural frequencies of human corticothalamic circuits. J Neurosci, 29, pp 7679-7685

Rossini PM, Barker AT, Berardelli A, Caramia MD, Caruso G, Cracco RQ, et al. (1994). Noninvasive electrical and magnetic stimulation of the brain, spinal cord and roots: basic principles and procedures for routine clinical application. Report of an IFCN committee. Electroencephalogr Clin Neurophysiol, 91, pp 79-92

Rossini PM, Rossi S. (2007). Transcranial magnetic stimulation: diagnostic, therapeutic, and research potential. Neurology, 68, pp 484-488

Rotenberg A. (2009). Prospects for clinical applications of transcranial magnetic stimulation and real-time EEG in epilepsy. Brain Topogr, 22, pp 257-266

Rothwell JC. (1997). Techniques and mechanisms of action of transcranial stimulation of the human motor cortex. J Neurosci Methods, 74, pp 113-122

Saglam M, Matsunaga K, Murayama N, Hayashida Y, Huang YZ, Nakanishi R. (2008). Parallel inhibition of cortico-muscular synchronization and cortico-spinal excitability by theta burst TMS in humans. Clin Neurophysiol, 119, pp 2829-2838

Salenius S, Portin K, Kajola M, Salmelin R, Hari R. (1997). Cortical control of human motoneuron firing during isometric contraction. J Neurophysiol, 77, pp 3401-3405

Sanger TD, Garg RR, Chen R. (2001). Interactions between two different inhibitory systems in the human motor cortex. J Physiol, 530, pp 307-317

Schutter DJ, Hortensius R. (2011). Brain oscillations and frequency-dependent modulation of cortical excitability. Brain Stimul, 4, pp 97-103

Siebner HR, Dressnandt J, Auer C, Conrad B. (1998). Continuous intrathecal baclofen infusions induced a marked increase of the transcranially evoked silent period in a patient with generalized dystonia. Muscle Nerve, 21, pp 1209-1212

Silvanto J, Cattaneo Z, Battelli L, Pascual-Leone A. (2008a). Baseline cortical excitability determines whether TMS disrupts or facilitates behavior. J Neurophysiol, 99, pp 2725-2730

Silvanto J, Muggleton N, Walsh V. (2008b). State-dependency in brain stimulation studies of perception and cognition. Trends Cogn Sci, 12, pp 447-454

Slotema CW, Blom JD, Hoek HW, Sommer IE. (2010). Should we expand the toolbox of psychiatric treatment methods to include Repetitive Transcranial Magnetic Stimulation (rTMS)? A meta-analysis of the efficacy of rTMS in psychiatric disorders. J Clin Psychiatry, 71, pp 873-884

Stefan K, Kunesch E, Benecke R, Cohen LG, Classen J. (2002). Mechanisms of enhancement of human motor cortex excitability induced by interventional paired associative stimulation. J Physiol, 543, pp 699-708

Stefan K, Kunesch E, Cohen LG, Benecke R, Classen J. (2000). Induction of plasticity in the human motor cortex by paired associative stimulation. Brain, $123 \mathrm{Pt} 3$, pp 572-584

Strafella AP, Valzania F, Nassetti SA, Tropeani A, Bisulli A, Santangelo M, et al. (2000). Effects of chronic levodopa and pergolide treatment on cortical excitability in patients with Parkinson's disease: a transcranial magnetic stimulation study. Clin Neurophysiol, 111, pp 1198-1202

Swartz BE, Goldensohn ES. (1998). Timeline of the history of EEG and associated fields. Electroencephalogr Clin Neurophysiol, 106, pp 173-176

Tallon-Baudry C, Bertrand O. (1999). Oscillatory gamma activity in humans and its role in object representation. Trends Cogn Sci, 3, pp 151-162

Tallon-Baudry C, Bertrand O, Delpuech C, Pernier J. (1996). Stimulus specificity of phaselocked and non-phase-locked $40 \mathrm{~Hz}$ visual responses in human. J Neurosci, 16, pp 4240-4249 
Tallon-Baudry C, Bertrand O, Peronnet F, Pernier J. (1998). Induced gamma-band activity during the delay of a visual short-term memory task in humans. J Neurosci, 18, pp 4244-4254

Thut G, Northoff G, Ives JR, Kamitani Y, Pfennig A, Kampmann F, et al. (2003). Effects of single-pulse transcranial magnetic stimulation (TMS) on functional brain activity: a combined event-related TMS and evoked potential study. Clin Neurophysiol, 114, pp 2071-2080

Thut G, Pascual-Leone A. (2010). A review of combined TMS-EEG studies to characterize lasting effects of repetitive TMS and assess their usefulness in cognitive and clinical neuroscience. Brain Topogr, 22, pp 219-232

Tokimura H, Di Lazzaro V, Tokimura Y, Oliviero A, Profice P, Insola A, et al. (2000). Short latency inhibition of human hand motor cortex by somatosensory input from the hand. J Physiol, 523 Pt 2, pp 503-513

Tsuji T, Rothwell JC. (2002). Long lasting effects of rTMS and associated peripheral sensory input on MEPs, SEPs and transcortical reflex excitability in humans. J Physiol, 540, pp 367-376

Udupa K, Ni Z, Gunraj C, Chen R. (2009). Interactions between short latency afferent inhibition and long interval intracortical inhibition. Exp Brain Res, 199, pp 177-183

Valls-Sole J, Pascual-Leone A, Wassermann EM, Hallett M. (1992). Human motor evoked responses to paired transcranial magnetic stimuli. Electroencephalogr Clin Neurophysiol, 85, pp 355-364

Van Der Werf YD, Paus T. (2006). The neural response to transcranial magnetic stimulation of the human motor cortex. I. Intracortical and cortico-cortical contributions. Exp Brain Res, 175, pp 231-245

Van Der Werf YD, Sadikot AF, Strafella AP, Paus T. (2006). The neural response to transcranial magnetic stimulation of the human motor cortex. II. Thalamocortical contributions. Exp Brain Res, 175, pp 246-255

Veniero D, Brignani D, Thut G, Miniussi C. (2011). Alpha-generation as basic responsesignature to transcranial magnetic stimulation (TMS) targeting the human resting motor cortex: A TMS/EEG co-registration study. Psychophysiology,

Voineskos AN, Farzan F, Barr MS, Lobaugh NJ, Mulsant BH, Chen R, et al. (2010). The role of the corpus callosum in transcranial magnetic stimulation induced interhemispheric signal propagation. Biol Psychiatry, 68, pp 825-831

von Stein A, Chiang C, Konig P. (2000). Top-down processing mediated by interareal synchronization. Proc Natl Acad Sci U S A, 97, pp 14748-14753

Wahl M, Lauterbach-Soon B, Hattingen E, Jung P, Singer O, Volz S, et al. (2007). Human motor corpus callosum: topography, somatotopy, and link between microstructure and function. J Neurosci, 27, pp 12132-12138

Wassermann EM, Lisanby SH. (2001). Therapeutic application of repetitive transcranial magnetic stimulation: a review. Clin Neurophysiol, 112, pp 1367-1377

Werhahn KJ, Kunesch E, Noachtar S, Benecke R, Classen J. (1999). Differential effects on motorcortical inhibition induced by blockade of GABA uptake in humans. J Physiol, 517 ( Pt 2), pp 591-597

Ziemann U. (2004). TMS and drugs. Clin Neurophysiol, 115, pp 1717-1729

Ziemann U, Rothwell JC. (2000). I-waves in motor cortex. J Clin Neurophysiol, 17, pp 397-405

Ziemann U, Rothwell JC, Ridding MC. (1996). Interaction between intracortical inhibition and facilitation in human motor cortex. J Physiol, 496 ( Pt 3), pp 873-881 


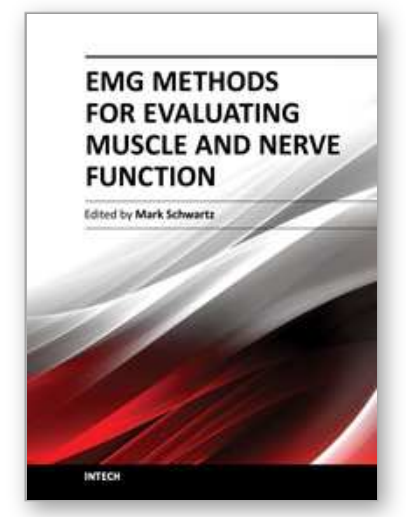

\author{
EMG Methods for Evaluating Muscle and Nerve Function \\ Edited by Mr. Mark Schwartz
}

ISBN 978-953-307-793-2

Hard cover, 532 pages

Publisher InTech

Published online 11, January, 2012

Published in print edition January, 2012

This first of two volumes on EMG (Electromyography) covers a wide range of subjects, from Principles and Methods, Signal Processing, Diagnostics, Evoked Potentials, to EMG in combination with other technologies and New Frontiers in Research and Technology. The authors vary in their approach to their subjects, from reviews of the field, to experimental studies with exciting new findings. The authors review the literature related to the use of surface electromyography (SEMG) parameters for measuring muscle function and fatigue to the limitations of different analysis and processing techniques. The final section on new frontiers in research and technology describes new applications where electromyography is employed as a means for humans to control electromechanical systems, water surface electromyography, scanning electromyography, EMG measures in orthodontic appliances, and in the ophthalmological field. These original approaches to the use of EMG measurement provide a bridge to the second volume on clinical applications of EMG.

\title{
How to reference
}

In order to correctly reference this scholarly work, feel free to copy and paste the following:

Faranak Farzan, Mera S. Barr, Paul B. Fitzgerald and Zafiris J. Daskalakis (2012). Combination of Transcranial Magnetic Stimulation with Electromyography and Electroencephalography: Application in Diagnosis of Neuropsychiatric Disorders, EMG Methods for Evaluating Muscle and Nerve Function, Mr. Mark Schwartz (Ed.), ISBN: 978-953-307-793-2, InTech, Available from: http://www.intechopen.com/books/emgmethods-for-evaluating-muscle-and-nerve-function/combination-of-transcranial-magnetic-stimulation-withelectromyography-and-electroencephalography-ap

\section{INTECH}

open science | open minds

\section{InTech Europe}

University Campus STeP Ri

Slavka Krautzeka 83/A

51000 Rijeka, Croatia

Phone: +385 (51) 770447

Fax: +385 (51) 686166

www.intechopen.com

\section{InTech China}

Unit 405, Office Block, Hotel Equatorial Shanghai

No.65, Yan An Road (West), Shanghai, 200040, China 中国上海市延安西路65号上海国际贵都大饭店办公楼 405 单元

Phone: +86-21-62489820

Fax: $+86-21-62489821$ 
(C) 2012 The Author(s). Licensee IntechOpen. This is an open access article distributed under the terms of the Creative Commons Attribution 3.0 License, which permits unrestricted use, distribution, and reproduction in any medium, provided the original work is properly cited. 